\title{
RESTOS FÓSILES DEL COCODRILO Diplocynodon (ALLIGATOROIDEA) EN EL MIOCENO INFERIOR DE LAS BARDENAS REALES DE NAVARRA
}

\author{
Xabier PEREDA SUBERBIOLA ${ }^{\prime .2}$, Xabier MURELAGA', \\ Humberto ASTIBIA' y Ainara BADIOLA'
}

\begin{abstract}
Universidad del País Vasco/Euskal Herriko Unibertsitatea, Facultad de Ciencias, Departamento de Estratigrafía y Paleontología, Apartado 644, 48080 Bilbao. E-mail: gpbmubej@lg.ehu.es

2 Muséum National d'Histoire Naturelle, Laboratoire de Paléontologie, 8 rue Buffon, 75005 París (Francia).
\end{abstract}

Pereda Suberbiola, X., Murelaga, X., Astibia, H. y Badiola, A. 2001. Restos fósiles del cocodrilo Diplocynodon (Alligatoroidea) en el Mioceno Inferior de las Bardenas Reales de Navarra. [Fossil remains of the crocodilian Diplocynodon (Alligatoroidea) from the Early Miocene of the Bardenas Reales of Navarre.] Revista Espainola de Paleontología, 16(2), 223-242. ISSN 0213-6937.

\begin{abstract}
A description of the first evidence on crocodilians from the Early Miocene (Tudela Formation, Ramblian, Biozones MN2b-3) of the Bardenas Reales of Navarre (NW Ebro Basin) is provided. The material consists of fragments of skull and lower jaw, teeth, vertebrae, appendicular bones, and osteoderms. Most are isolated elements, disarticulated, and belong to several individuals, either juveniles or adults. The low morphological variation observed in this fossil association suggests that all remains belong to a single taxon. The material is referre to Diplocynodon sp. (Eusuchia, Alligatoroidea), mainly on the basis of the occurrence of alveoli for dentary teeth 3 and 4 nearly of same size and confluent, splenial excluded from mandibular symphysis, and ventral osteoderms which consist of paire ossifications. The lower jaws are characterise by a very short symphysis, which does not extend beyond the posterior edge of the alveolus of the tooth 3 . The localities of the Bardenas Reales of Navarre have yielded the most abundant and diverse crocodilian assemblage never described from the Miocene of the Iberian Peninsula.
\end{abstract}

Keywords: Crocodylia, Diplocynodon, Early Miocene, Ebro Basin, Iberian Peninsula.

\section{RESUMEN}

Se describen por primera vez restos fósiles de cocodrilo del Mioceno Inferior (Formación Tudela, Rambliense, Biozonas MN2b-3) de las Bardenas Reales de Navarra, región situada en el sector noroccidental de la Depresión del Ebro. El material consiste en fragmentos craneales y mandibulares, dientes, huesos del esqueleto axial y apendicular y del escudo dérmico. La mayor parte de los restos están desarticulados y pertenecen a varios individuos, habiéndose reconocido tanto formas juveniles como adultas. La escasa variabilidad morfológica observada en la asociación fósil sugiere que todos los restos pertenecen al mismo taxón. El material se atribuye a Diplocynodon sp. (Eusuchia, Alligatoroidea). Dicha atribución se basa en caracteres mandibulares, como la presencia de alveolos dentarios tercero y cuarto del mismo tamaño y confluentes, esplenial excluido de la sínfisis mandibular, y en rasgos de los osteodermos ventrales, que están formados por osificaciones pares. La principal característica de los dentarios es una sínfisis mandibular muy corta, que no sobrepasa en longitud el borde posterior del tercer alveolo. Los yacimientos de las Bardenas Reales de Navarra han proporcionado el material más abundante y diverso de Diplocynodon conocido en el Mioceno de la Península Ibérica.

Palabras clave: Crocodylia, Diplocynodon, Mioceno Inferior, Depresión del Ebro, Península Ibérica. 


\section{ENGLISH ABRIDGED VERSION}

The region of the Bardenas Reales is located in the southwestern part of Navarre. Geologically, it is situated on the northwestern area of the Cenozoic Ebro Basin (Fig. 1), which is mainly composed of alluvial, lacustrine and palustrine deposits of Middle Eocene to Late Miocene age. As compared to other areas of the Ebro Basin, the Bardenas has been poorly studied from a palaeontological point of view. The deposits of Early Miocene age (Ramblian-Aragonian) which outcrop in the Bardenas Reales belong to the Tudela Formation. They consist of clays, sandstones, marls and limestones, with some intercalations of gypsum. Most of the vertebrate fossils have been found in blue to grey marls. The micromammal association found in the Bardenas sites suggests that the fossiliferous deposits belong to the biozones Z-A of the Ramblian (sensu Daams and Freudenthal, 1990; see Astibia et al., 1995; Murelaga, 2000), that is, to the late Aquitanian and early Burdigalian. They are approximately equivalent to the biozones MN2b-3 of the European Neogene (Mein, 1990).

The vertebrate fauna of the Bardenas consists of teleost fish, amphibians, lizards, snakes, amphisbaenians, crocodilians, turtles, birds, and mammals (insectivores, chiropters, rodents, lagomorphs, artiodactyls and perissodactyls) (Murelaga, 2000). Crocodilian remains are relatively abundant and are known in a number of localities (BCo, BF1, BF2, BT1, BT2, BT3, BT4, BT5, BTr, CJ, CV, N1, N2a, N2b, N3, N4, N5a, N5b, RB). The material consists of fragments of skull and lower jaw, teeth, vertebrae, appendicular bones, and osteoderms (Figs. 2-5). Most of the crocodilian remains are disarticulated and have been collected as isolated bones and teeth. The low morphological variation observed in the fossil association suggests that all remains belong to a single taxon, being either immature or adult specimens. The crocodilian material from the Bardenas was previously referred to as cf. Diplocynodon sp. by Pereda Suberbiola et al. (1996). A large surangular fragment (Fig. 3o) is similar in size to that of the specimen MNHN SG 541 of Diplocynodon rateli kept in Paris (lower jaw length $40 \mathrm{~cm}$ ) and probably belongs to an individual of about $3 \mathrm{~m}$ long. Some of the smallest remains (e.g. vertebrae and teeth) are presumably from juveniles less than $1 \mathrm{~m}$ long.

The specimen BF2-2 consists of skull (frontal, quadrate, quadratojugal, pterygoid and maxilla, but remains from the orbital and postorbital regions could as well be represented) and lower jaw remains, as well as teeth, the odontoid process of the axis and osteoderms (Figs. 3a-e, 4a-b, 5a). All they belong to a single adult. Other isolated skull bones from different localities include fragments of maxilla, frontal, postorbital, squamosal, jugal and exoccipital (Figs. 3f-j, 4c). We attempt a partial reconstruction of the mandible based on the evidence of dentary (see measurements in Fig. 6), angular, surangular and articular fragments (Figs. 3k-q, $4 d-f)$. Isolated teeth are very abundant in the Bardenas localities (Fig. 3r). The vertebral column is represented by cervical, dorsal and caudal vertebrae from several individuals (Figs. 5b-h). The appendicular skeleton includes only hindlimb bones (femur, tibia) (Figs. 5i-k). Finally, the dermal skeleton is represented by abundant scutes (Figs. 5l-p).

The main features of the Bardenas crocodilian are as follows: frontal participates posterolaterally in the supratemporal fossa and prevents broad contact between the postorbital and parietal; foramen aerum located on the dorsal surface of the quadrate ramus; alveoli for dentary teeth 3 and 4 are nearly of same size and confluent; very short mandibular symphysis, which does not extend beyond the $3^{\text {rd }}$ alveolus; splenial excluded from the mandibular symphysis; the mark of the splenial above and under the Meckelian groove indicates that the ventral tip of the splenial is more projected near the symphysis (up to the $4^{\text {th }}$ alveolus) than the dorsal tip (up to $5^{\text {th }}$ or $6^{\text {th }}$ alveolus); conical teeth, oval to subcircular in cross section; crowns ornamented with fine longitudinal ridges, slightly anastomosed towards the apex; procoelous presacral and caudal vertebrae; hypapophysis absent in the first postaxial vertebra; rectangular dorsal osteoderms with a longitudinal keel; ventral armour consisting of paired ossifications that suture together, the anterior ossification narrow and with a smooth area, while the posterior one is fully ornamented.

The presence of procoelous vertebrae is a synapomorphy of the Eusuchia, although it is paralleled in other crocodilians (Benton and Clark, 1988; Michard et al., 1990; Clark, 1994). Prominent hypapophyses on cervical centra occur in eusuchians (poorly developed in Gavialis), but also in Bernissartia (poorly developed) and dyrosaurids (Norell and Clark, 1990). The foramen aerum on the dorsal surface of the quadrate is a derived character of the Alligatoroidea (Brochu, 1999). The Bardenas crocodilian shares with the Diplocynodontinae of Brochu (1999), a clade composed of Diplocynodon and Baryphracta, the occurrence of paired ossifications on the ventral armour. The basal eusuchian Borealosuchus may show this character as well, but it is considered as due to convergence (Brochu, 1997, 1999). The Bardenas crocodilian shares with the known species of Diplocynodon the absence of the splenial from the symphysis (Buscalioni et al., 1992). A splenial symphysis is present ancestrally in Alligatoroidea (see Brochu, 1999). This condition is retained by an unpublished alligatoroid from the Late Paleocene of Cernay-Mont-Berru (Champagne, France), here provisionally referred to aff. Diplocynodon sp. (Lapparent de Broin, com. pers.), and suggests that its absence could have occurred at least twice among Alligatoroidea, in Alligatoridae and in Diplocynodontinae (sensu Brochu, 1999).

Moreover, the Bardenas alligatoroid has confluent third and fourth dentary alveoli, as Diplocynodon. Rauhe and Rossmann (1995) claimed that this character is a synapomorphy of the Leidyosuchidae (Diplocynodon + Leidyosuchus), but Buscalioni et al. (1992) regarded it as plesiomorphic character state. Brochu (1999) considered 
it either as a plesiomorphic character state or as an ambiguous character of Diplocynodon. Indeed, its presence requires an identical autapomorphic transformation in Leidyosuchus canadensis. Confluent third and fourth dentary alveoli are also known in Bernissartia and Borealosuchus (Brochu, 1999: fig. 34). The Globidonta of Brochu (1999), i.e. all alligatoroids except the Diplocynodontinae, have separated third and fourth dentary alveoli. Frey et al. (1987) suggested separation of the third and fourth alveoli in Baryphracta, but as all specimens known are preserved with the jaws closed, this region is not available (Brochu, 1999).

The Bardenas dentaries differ from those of species of Diplocynodon in having a shorter mandibular symphysis, which does not extend beyond the third alveolus (Fig. 6). In Diplocynodon, the symphysis length is variable according to the species and extends from the third to fifth alveoli (Brinkmann and Rauhe, 1998). The symphysis length seems to be related to size in Alligator mississipiensis (see Brochu, 2000), but this is not the rule in Diplocynodon and closely allied forms. Pending a fully revision of the European material, the Bardenas crocodilian is referred to Diplocynodon sp. indet.

Brochu (1999) defined the Diplocynodontinae as "a stem-based monophyletic group including Diplocynodon rateli and all crocodylians more closely related to it than to Alligator mississippiensis". His phylogenetic work supports the monophyly of Diplocynodontinae. However, previous works have raised questions regarding the monophyly of Diplocynodon (Buscalioni et al., 1992). A global analysis including all known species of Diplocynodon, Baryphracta deponiae and other basal alligatorids is needed to resolve the problem.

From a palaeoecological point of view, the Diplocynodon from the Bardenas was an inhabitant of palustrine or lacustrine environments. It takes part of the para-autochthonous assemblage of the fossil association (aquatic forms). There is no differences in the reptile faunal composition of the Bardenas sites. This suggests that the climatic changes between the biozones $\mathrm{Z}$ and $\mathrm{A}$ of the Ramblian, as supported by the study of mammal cenograms (Murelaga, 2000) and of ostracods (Murelaga et al., 1997), were not significant enough (i.e., temperature fall, change in dryness) to have an ecological effect on the reptiles such as turtles and crocodilians (Diplocynodon).

\section{INTRODUCCIÓN}

En comparación con el registro fósil del Paleógeno, los restos fósiles de cocodrilos son relativamente escasos (con la excepción de dientes aislados) en las formaciones de edad Mioceno de la Península Ibérica (Buscalioni, 1986b). No obstante, los cocodrilos son uno de los grupos de vertebrados mejor representados en los depósitos continentales del Mioceno Inferior de las Bardenas Reales y zonas adyacentes de Navarra. La primera cita sobre el hallazgo de cocodrilos en el área de Tudela es de 1966, fecha en la que Crusafont, Truyols y Riba dieron a conocer dos niveles fosilíferos (datados como de edad Ageniense) que denominaron Tudela I y II. El material de cocodrilo citado por Crusafont et al. (1966), un cráneo fragmentario de un posible aligatoroideo, está en proceso de restauración y estudio en la Universidad Autónoma de Madrid (F. Ortega, com. pers.). Astibia et al. (1995) describieron nuevas localidades de vertebrados fósiles en la comarca de las Bardenas Reales de Navarra, mencionando la presencia de restos de estos arcosaurios. Este material se asignó de manera preliminar a cf. Diplocynodon sp. (Pereda Suberbiola et al., 1996).

La fauna de vertebrados continentales del Mioceno Inferior de las Bardenas está compuesta por peces teleósteos, anfibios anuros y urodelos, lagartos, serpientes, anfisbenas, cocodrilos, tortugas (Murelaga et al., 1999), aves caradriformes y mamíferos insectívoros, quirópteros, roedores, lagomorfos, artiodáctilos y perisodáctilos (Murelaga, 2000). En este trabajo se describen los restos de cocodrilos descubiertos hasta la fecha, discutiéndose sus afinidades sistemáticas e interés paleobiológico.

Buscalioni (1986b) asignó provisionalmente al género Diplocynodon material proveniente del Mioceno Inferior de Fuenmayor (La Rioja) y del Mioceno Superior de Tejares de Jun (Granada). A estos restos hay que añadir el cráneo incompleto mencionado por Crusafont et al. (1966) en el Mioceno Inferior de Tudela (Navarra). Otro material del Mioceno ibérico consiste en restos de cocodrilo indeterminado hallados en el Burdigaliense superior de Gelida (Barcelona) y el Mioceno Superior de Venta del Moro (Valencia) (Buscalioni, 1986b). El material de las Bardenas de Navarra representa la asociación fósil de Diplocynodon más diversa conocida hoy en día en el Mioceno de la Península ibérica y la única estudiada en detalle hasta la fecha.

\section{MARCO GEOGRÁFICO Y GEOLÓGICO}

La comarca de las Bardenas Reales se localiza en el extremo sudeste de la Comunidad Foral de Navarra (Fig. 1). Se ubica en el borde noroccidental de la cuenca cenozoica continental del Ebro, que está constituida principalmente por depósitos aluviales, lacustres y palustres de edad Eoceno Medio-Mioceno Superior. Se trata de una región muy poco estudiada desde un punto de vista paleontológico, a diferencia de otras áreas colindantes (Cuenca et al., 1992).

Los materiales del Mioceno Inferior que afloran en las Bardenas Reales de Navarra pertenecen a la Formación Tudela. Consisten principalmente en arcillas, areniscas, margas y calizas, con algunas intercalaciones de yesos. La Formación Tudela es de edad RamblienseAragoniense (Crusafont et al. 1966; Astibia et al., 1995). Desde un punto de vista litológico, está constituida principalmente por arcillas de tonalidades rojas y ocres, margas grises y beiges, entre las cuales se intercalan capas de calizas, areniscas y yesos. Esta formación se interpreta como el relleno sedimentario de la parte central de la Cuenca Navarro-Riojana durante el Rambliense y el 


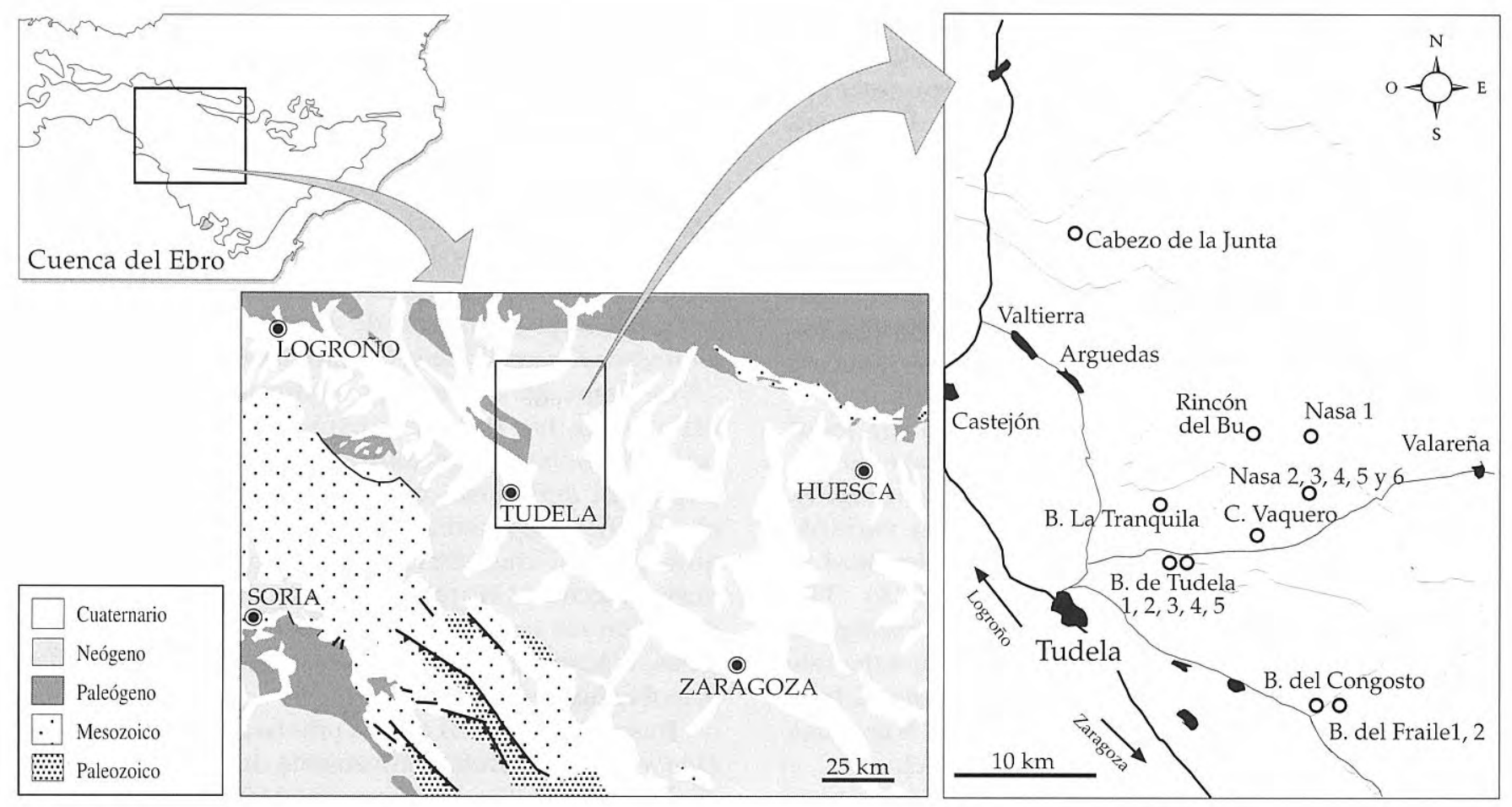

Figura 1. Mapa de localización geográfica de los yacimientos del Mioceno Inferior de las Bardenas Reales de Navarra que han proporcionado restos fósiles de cocodrilos (modificado de Azanza, 1986).

Location map of the Early Miocene sites of the Bardenas Reales of Navarre that have yielded crocodilian remains (modified from Azanza, 1986).

Aragoniense por facies aluviales distales, provenientes de los márgenes elevados de los Pirineos y de la Ibérica, y de facies palustres o lacustres.

La mayor parte de los restos de vertebrados se han hallado en margas gris-azuladas. La asociación de micromamíferos indica que los yacimientos fosilíferos se inscriben en las biozonas $\mathrm{Z}$ y A del Rambliense (sensu Daams y Freudenthal, 1990), equivalentes a la escala europea del Aquitaniense superior y Burdigaliense inferior (Murelaga, 2000).

Las localidades de las Bardenas que han proporcionado restos de cocodrilos son: Barranco del Congosto (BCo), Barranco del Fraile 1 (BF1) y 2 (BF2), Barranco de Tudela 1 (BT1), 2 (BT2), 3 (BT3), 4 (BT4) y 5 (BT5), Barranco de la Tranquila (BTr), Cabezo de la Junta (CJ), Cabezo Vaquero (CV), La Nasa 2 (N2a y N2b), 3 (N3), 4 (N4), 5 (N5) y 6 (N6), Rincón del Bu (RB) y Tripazul (= La Nasa 1) (N1) (Murelaga, 2000) (Fig. 1). Barranco del Fraile y Barranco de Tudela 3 son los yacimientos que han proporcionado la mayor cantidad de restos. El yacimiento más septentrional es el del Cabezo de la Junta, cuya asociación de micromamíferos corresponde a la biozona Z. Los niveles fosilíferos más meridionales, que incluyen las demás localidades, pertenecen a la biozona $\mathrm{A}$, debido principalmente a la total ausencia de cricétidos (Astibia et al., 1995; Murelaga, 2000). Estas dos biozonas del Rambliense, Z y A, corresponden en parte a las biozonas MN2b y MN3 del Neógeno europeo (Mein, 1990).

\section{MATERIAL ESTUDIADO}

El material de cocodrilo de las Bardenas Reales de Navarra consiste en restos craneales y mandibulares, dientes, vértebras, huesos de las extremidades y osteodermos (Figs. 2-5). Los restos fósiles, depositados provisionalmente durante su estudio en el Laboratorio de Paleontología de la Universidad del País Vasco/EHU de Bilbao, forman parte de las Colecciones Paleontológicas del Museo de Navarra. Muchos de los fósiles han sido recogidos en superficie, pero también hay microrrestos (principalmente dientes aislados), obtenidos mediante el lavado y tamizado del sedimento. La mayor parte de los restos están desarticulados y corresponden a varios individuos, estando representados diferentes estadios de crecimiento ontogenético. La variabilidad morfológica observada en los elementos de la asociación fósil es poco significativa, lo que nos lleva a asignar todo el material al mismo taxón: Diplocynodon sp.

Se ha comparado el material fósil de las Bardenas con el de varias especies de Diplocynodon y aff. Diplocynodon sp. conservado en las colecciones paleontológicas del Museum National d'Histoire Naturelle (MNHN) de París, Francia. La lista de taxa y material de comparación es la siguiente:

- Diplocynodon rateli Pomel, 1847 (especie tipo) del Mioceno Inferior (MN 2a) de Saint-Gérand-le-Puy, Departamento del Allier, Auvernia (10 especímenes: MNHN SG $497,498,499,500,520,543,548,549,551$ y 12853 );

- Diplocynodon styriacus (Hofmann, 1885) del Mioceno Inferior (MN 4a) de Artenay, Departamento del Loiret, 
Orléanais (6 especímenes, MNHN ART 360, 1367, 1961, 6222,6321 y sin catalogar);

- Diplocynodon sp. del Eoceno Inferior (MP 10) de Mancy, Departamento del Marne, Champaña (material inédito: 4 especímenes, MNHN MA 15413, 15414, 15416 y sin catalogar);

- aff. Diplocynodon sp. del Paleoceno superior (MP 6) de Cernay-Mont-Berru, Departamento del Allier, Champaña (material inédito: 5 especímenes, MNHN BR 2617, 4021, 13217, 15198 y CRL 13098). Su atribución sistemática es provisional, en tanto este material está aún por publicar (Lapparent de Broin, com. pers.).

También se han hecho comparaciones con el material de Diplocynodon y formas afines descrito en la literatura:

- Diplocynodon rateli Pomel, 1847 (referencias: Berg, 1966; Brinkmann y Rauhe, 1998; Brochu, 1999)

- D. tormis Buscalioni, Sanz y Casanovas, 1992 (referencia: Buscalioni et al., 1992)

- D. styriacus (Hofmann, 1885) de Francia (referencia: Ginsburg y Bulot, 1997)

- D. hantoniensis (Wood, 1846) (referencias: Vignaud et al., 1996; Brochu, 1999)

- D. darwini (Ludwig, 1877) (referencia: Berg, 1966)

- Baryphracta deponiae Frey, Laemmert y Riess, 1987 (referencia: Frey et al., 1987).

Por último, se ha utilizado la osteología de los cocodrilos actuales Crocodylus niloticus, Caiman crocodilus y Alligator mississippiensis como patrón de referencia (colecciones de Anatomie Comparée y de Paléontologie, MNHN de París).

\section{PALEONTOLOGíA SISTEMÁtiCA}

\author{
Eusuchia Huxley, 1875 \\ Alligatoroidea Gray, 1844 (sensu Norell et al., 1994) \\ Diplocynodon Pomel, 1847
}

Especie tipo: Diplocynodon rateli Pomel, 1847.

\section{Diplocynodon sp. Figs. 3-5}

Sinonimias: cf. Diplocynodon sp. (Astibia et al., 1995; Pereda Suberbiola et al. 1996).

\section{Material}

BF2-2, fragmentos craneales (frontal, cuadrado, cuadratoyugal, pterigoides, maxilar), mandibulares (dentario, angular, articular), dientes asociados, proceso odontoides del axis y restos de osteodermos. Material craneal aislado: fragmentos de maxilar (BF-53, BT2-14, N122, N3-12), frontal (N2-26), postorbital (N2-11), escamoso (N5-12, N1-62), yugal (CJ-7) y exoccipital (N1-7). Material mandibular aislado: restos de dentario (BF-38, BF-46, N230, BT3-182), angular (N5-26), articular (RB1-4) y suprangular (BT3-183, N2-18). Dientes aislados (BF-47, BF-49, BF-71, CJ-9, N5-30, RB sin catalogar). Material postcraneal: vértebras cervicales (BF-91, BT3-3, BT3-6, BT3-9, BT3-10, BT3-61, BT3-69, BT3-72, N1-39, N2-67,
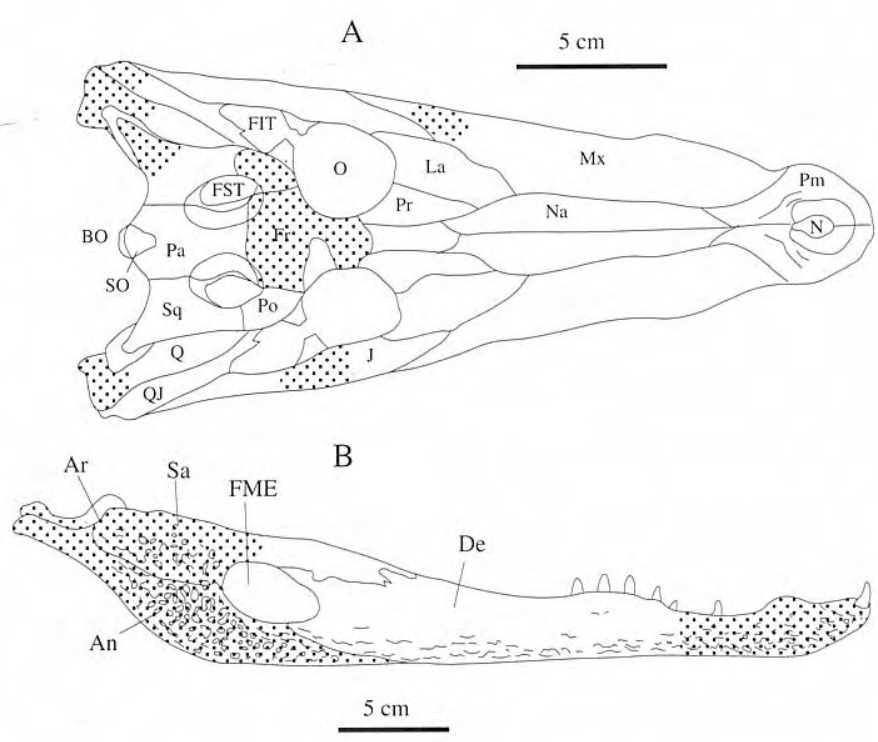

Figura 2. A, esquema del cráneo de Diplocynodon en vista dorsal (basado en D. tormis según Buscalioni et al., 1992). B, esquema de la mandíbula de Diplocynodon en vista lateral (basado en $D$. styriacus según Ginsburg y Bulot, 1997). Las zonas sombreadas corresponden al material fósil descubierto en las Bardenas de Navarra. Leyenda: Cráneo - Bo, basioccipital; FIT, fosa infratemporal; Fr, frontal; FST, fosa supratemporal; J, yugal; La, lacrimal; Mx, maxilar; $\mathrm{N}$, narinas externas; $\mathrm{Na}$, nasal; $\mathrm{O}$, órbita; $\mathrm{Pa}$, parietal; $\mathrm{Pm}$, premaxilar; $\mathrm{Po}$, postorbital; Pr, prefrontal; Q, cuadrado; Qj, cuadratoyugal; So, supraoccipital; Sq, escamoso. Mandíbula - An, angular; Ar, articular; De, dentario; FME, fosa mandibular externa; Sa, suprangular.

A, schema of the skull of Diplocynodon in dorsal view (based on D. tormis from Buscalioni et al., 1992). B, schema of the lower jaw of Diplocynodon in lateral view (based on D. styriacus from Ginsburg and Bulot, 1997). The dotted areas represent fossil remains found in the Bardenas of Navarre. Key: Skull - Bo, basioccipital; FIT, infratemporal fenestra; Fr, frontal; FST, supratemporal fenestra; J, jugal; La, lacrimal; $M x$, maxilla; $N$, external nares; $\mathrm{Na}$, nasal; $O$, orbit; $P a$, parietal; Pm, premaxilla; Po, postorbital; Pr, prefrontal; $Q$, quadrate; $Q j$, quadratojugal; So, supraoccipital; Sq, squamosal. Mandible - An, angular; Ar, articular; De, dentary; FME, external mandibular fenestra; Sa, suprangular.

N3-9, N4-13), vértebras de la transición cervicotorácica (BF-48, N1-18), vértebras dorsales (BF-17, BF-18, BF-22, BF-28, BF-56, BT3-63, BT3-64, BT3-65, ВT3-67, ВT3-68, ВТ3-76, ВТ3-77, ВТ3-78, ВТ3-177, ВТ3-178, ВТ3-179, BT3-180, RB1-7) y caudales (BF-27, BF-31, BF-75, BT3-2, BT3-181, N5-7), otros restos vertebrales (BF-66, BF-92, 
BF-81, BF-82, BT3-4, BT3-16, N1-80, N3-6, N3-44, RB1$15)$, elementos del esqueleto apendicular (BF-32, BF-51, BF-99, BT3-8, BT3-62, N1-25, N6-8) y osteodermos (BF24, BF-35, BF-37, BF-45, BF-50, BF-57, BF-59, BF-67, BF-70, BF-73, BF-77, BF-83, BF-86, BF-90, BF-93, BF162, BF-163, BT1-1, BT2-5, BT2-7, BT2-11, BT2-13, ВT31, ВT3-5, ВT3-7, ВТ3-12, ВT3-14, ВT3-15, ВТ3-18, ВТ319, ВТ3-20, ВТ3-168, ВТ3-171, ВТ3-172, ВТ3-173, ВТ3174, ВT3-175, BT5-1, ВT5-3, ВT5-8, ВT5-9, BTr-27, BTr31, BTr-34, CJ-10, CJ-2, CJ-3, CJ-156, CV-21, CV-30, N120, N1-21, N1-23, N1-24, N1-26, N1-27, N1-28, N1-29, N1$44, \mathrm{~N} 1-71, \mathrm{~N} 1-81, \mathrm{~N} 1-82, \mathrm{~N} 2-49$, N2-50, N2-52, N2-57, N258 , N2-59, N2-60, N2-63, N2-64, N2-65, N2b-1, N3-4, N35, N3-20, N3-21, N3-29, N3-31, N3-32, N3-33, N3-40, N353, N3-54, N3-77, N4-4, N4-6, N4-7, N4-10, N5-13, N5-15, N5-18, N5-20, RB1-18, RB1-40, RB1-42, RB1-47).

\section{DESCRIPCIÓN}

El material incluye numerosos restos aislados y desarticulados, principalmente dientes, vértebras y osteodermos. También hay material fragmentario muy meteorizado que se encontró en superficie en conexión anatómica, del que se conservan restos craneales y postcraneales (espécimen BF2-2). Se interpreta que los ejemplares de pequeño tamaño corresponden a individuos juveniles con una longitud total inferior a $1 \mathrm{~m}$, mientras que los restos de mayor tamaño corresponderían a individuos adultos de hasta $3 \mathrm{~m}$ de longitud.

\section{Cráneo y mandíbula (Fig. 2)}

- Fragmentos craneales y mandibulares del individuo BF2-2 (Figs. 3a-e, 4a-b)

Se han podido reconocer los siguientes huesos: frontal, cuadrado, cuadratoyugal, pterigoides y fragmentos del maxilar. También pueden estar representados otros restos de la región orbital y postorbital, pero el material es muy fragmentario para hacer una identificación fiable. Los restos mandibulares incluyen fragmentos del angular, articular y, probablemente, del dentario. Los dientes son fragmentarios y pueden pertenecer al maxilar o a la mandíbula.

Frontal (Fig. 3a, 4a). El frontal está roto a la altura del puente transverso interorbital, de tal modo que no se conserva el proceso anterior. En esta zona, la superficie de sutura con el prefrontal es visible. La superficie dorsal es plana y presenta una ornamentación formada por alveolos ovales dispuestos de manera regular en la región interorbital. Los bordes de la órbita no están elevados con respecto al espacio interorbital. Los márgenes posterolaterales del frontal participan en la construcción del borde anterior de la fosa supratemporal. La superficie de sutura con el parietal tiende a ser rectilínea. El frontal es de un tamaño comparable al del individuo MNHN SG 539 de Diplocynodon rateli de Saint-Gérand-Le Puy (Allier, Auvernia), lo que indica una longitud craneal aproximada de $25 \mathrm{~cm}$.

Cuadrado (Fig. 3c, 4b). Se conservan las extremidades distales de los cuadrados izquierdo y derecho. Ambas conservan los cóndilos para la articulación con la mandíbula. El cóndilo lateral está más desarrollado que el medial. El foramen aerum no está situado en posición medial de la rama articular del cuadrado sino en posición ligeramente lateral.

Cuadratoyugal (Fig. 3b). Un fragmento de cuadratoyugal se articula con el cuadrado izquierdo. En vista ventral, el cuadratoyugal es ligeramente cóncavo y presenta una superficie medial de sutura para el cuadrado y una superficie lateral de sutura para el yugal. Tal y como se conserva, la superficie de sutura con el cuadrado es rectilínea. A diferencia del cuadrado, la superficie dorsal del cuadratoyugal presenta una ornamentación con alveolos subcirculares u ovales de tamaño variable. El cuadratoyugal no interviene en la articulación mandibular.

Figura 3. Restos craneales y mandibulares de Diplocynodon sp., Mioceno Inferior de las Bardenas Reales de Navarra; a-e, BF2-2: a, frontal en vistas dorsal (a1) y ventral (a2); b, cuadratoyugal izquierdo en vistas dorsal (b1) y ventral (b2); c, cuadrado izquierdo en vistas dorsal (c1) y posterior (c2); d, pterigoides derecho en vista ventral; e, articular izquierdo en vista dorsal; f-j, restos craneales aislados: f, N2-26, frontal en vista dorsal; g, N2-11, postorbital izquierdo en vista dorsal; h, N1-62, escamoso izquierdo en vista dorsal; i, N5-12, escamoso izquierdo en vista dorsal; j, N1-7, exoccipital en vista posterior; k-q, restos mandibulares aislados: k, N2-30, dentario izquierdo en vistas dorsal $(\mathrm{k} 1)$, medial $(\mathrm{k} 2)$ y lateral $(\mathrm{k} 3)$; 1, BF-38, dentario derecho en vistas dorsal (11), medial (12) y lateral (13); m, BF-46, dentario derecho en vistas dorsal (m1), medial (m2) y lateral (m3); n, BT3-182, fragmento de dentario izquierdo en vista dorsal; o, BT-183, suprangular derecho en vista lateral; p, N2-18, suprangular derecho en vista lateral; q, N5-26, angular izquierdo en vistas lateral (q1) y medial (q2); r, BF-47, diente en vistas lingual (r1) y mesial o distal (r2). Escala: $2 \mathrm{~cm}$ (fotografías a tamaño natural).

Skull and lower jaw remains of Diplocynodon sp., Early Miocene of the Bardenas Reales of Navarre; a-e, BF2-2: a, frontal in dorsal (a1) and ventral (a2) views; b, left quadratojugal in dorsal (b1) and ventral (b2) views; c, left quadrate in dorsal ( $c 1)$ and posterior (c2) views; $d$, right pterygoid in ventral view; e, left articular in dorsal view; $f$-j, isolated skull remains: $f, N 2-26$, frontal in dorsal view; $g, N 2-11$, left postorbital in dorsal view; h, N1-62, left squamosal in dorsal view; $i, N 5-12$, left squamosal in dorsal view; $j, N 1-7$, exoccipital in posterior view; $k-q$, isolated mandibular remains: $k, N 2-30$, left dentary in dorsal ( $k 1)$, medial $(k 2)$ and lateral $(k 3)$ views;, , BF-38, right dentary in dorsal (l1), medial (l2) and lateral (l3) views; $m, B F-46$, right dentary in dorsal (m1), medial (m2) and lateral (m3) views; $n$, BT3-182, fragment of left dentary in dorsal view; o, BT-183, right surangular in lateral view; $p, N 2-18$, right surangular in lateral view; $q, N 5-26$, left angular in lateral (q1) and medial (q2) views; $\boldsymbol{r}, B F-$ 47 tooth in lingual (r1) and mesial or distal (r2) views. Scale: $2 \mathrm{~cm}$ (photographs at natural size). 


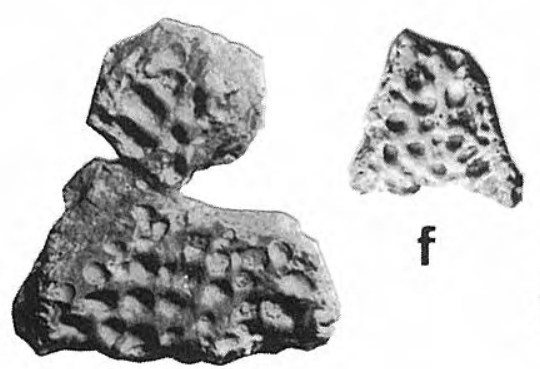

$a_{1}$
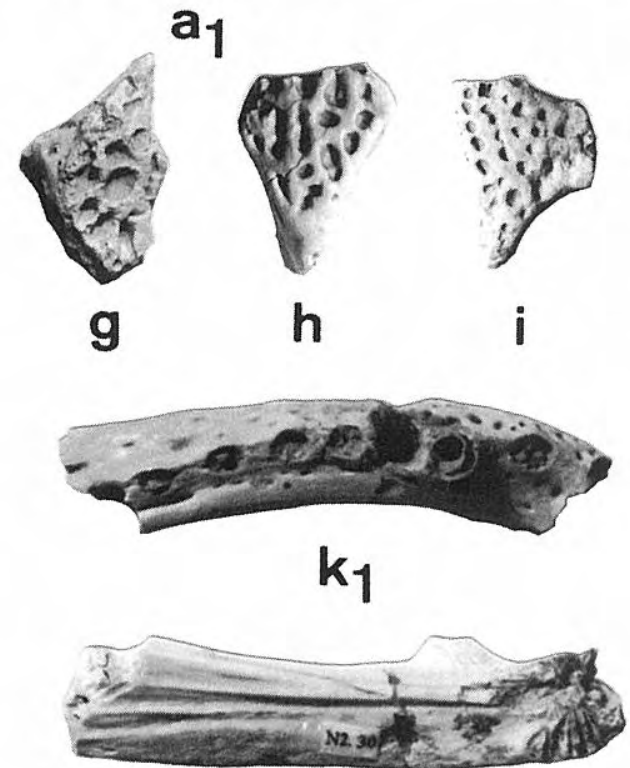

$k_{2}$

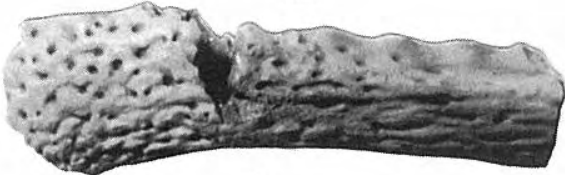

$k_{3}$
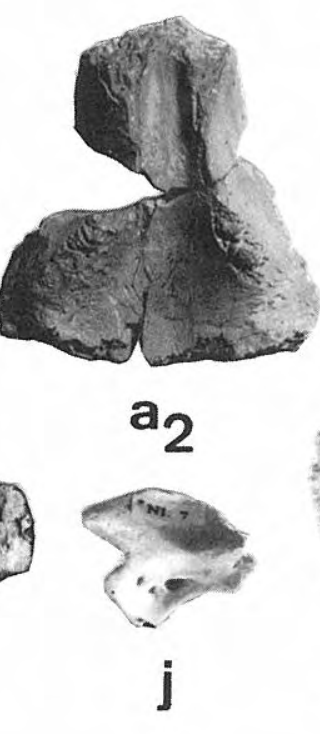
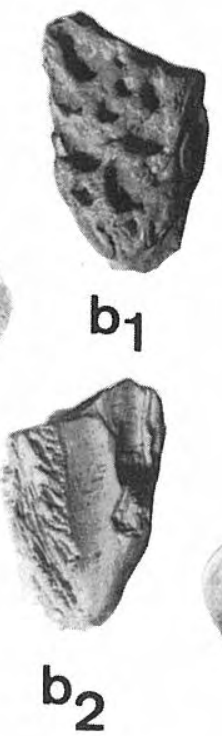
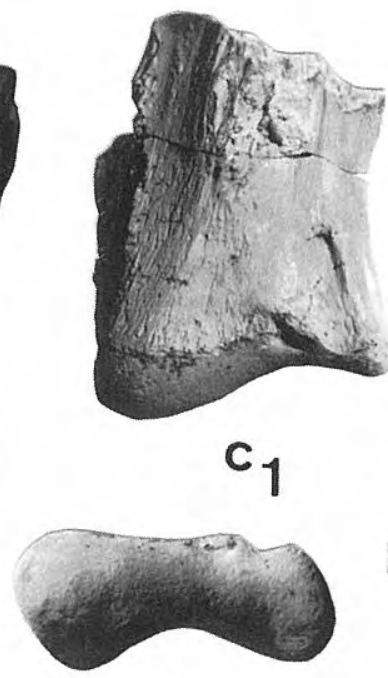

$c_{2}$
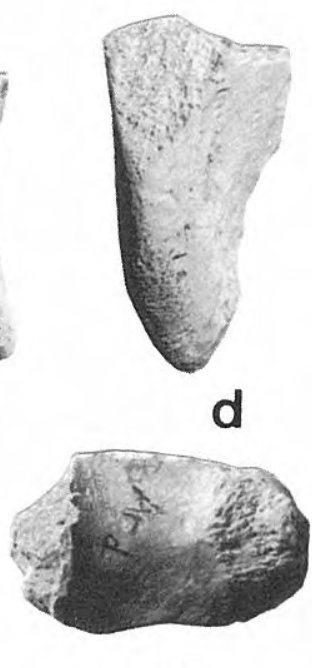

e
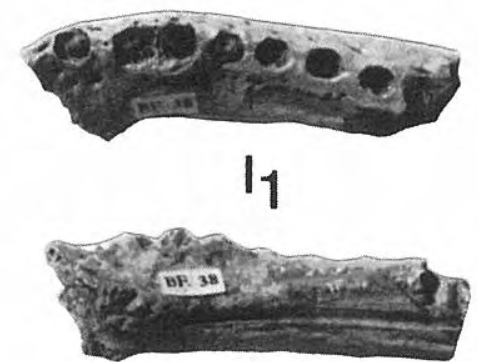

$I_{2}$

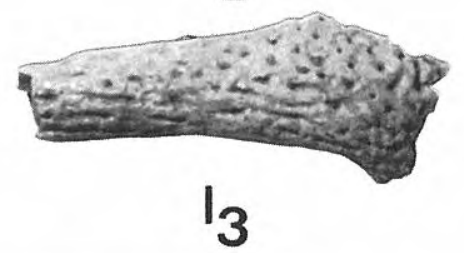

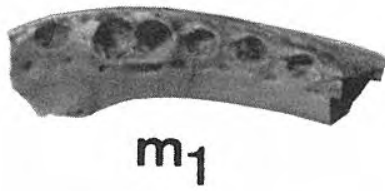

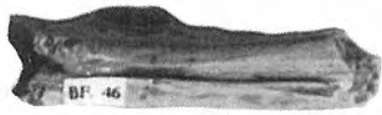

$m_{2}$
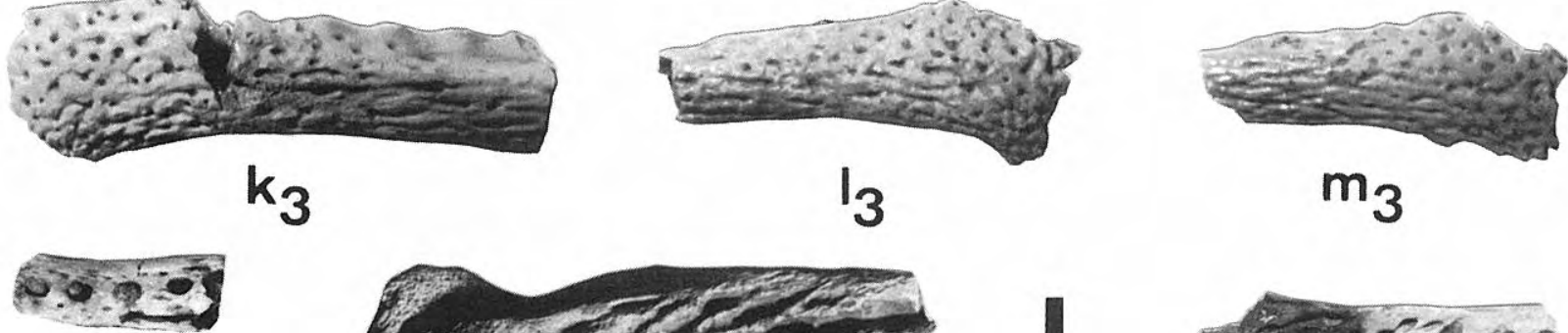

n
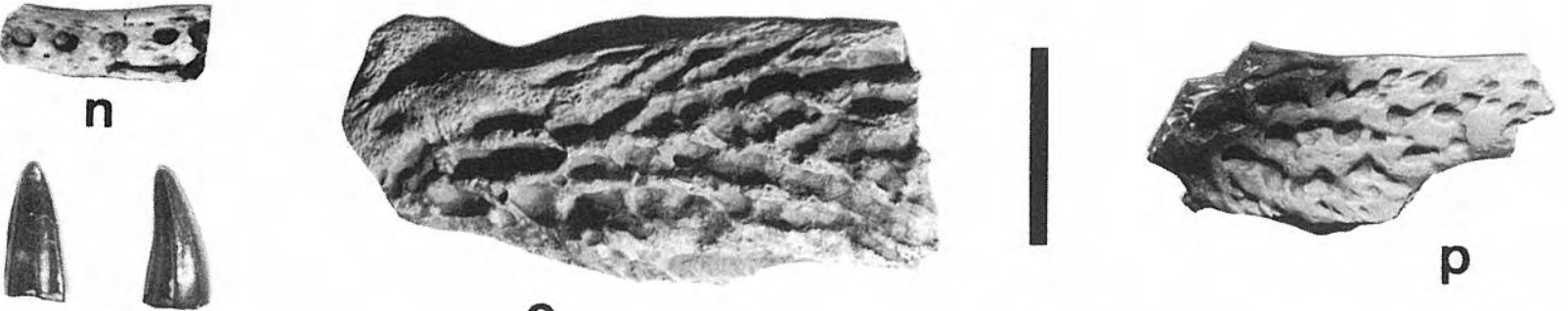

O

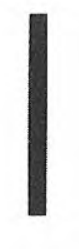

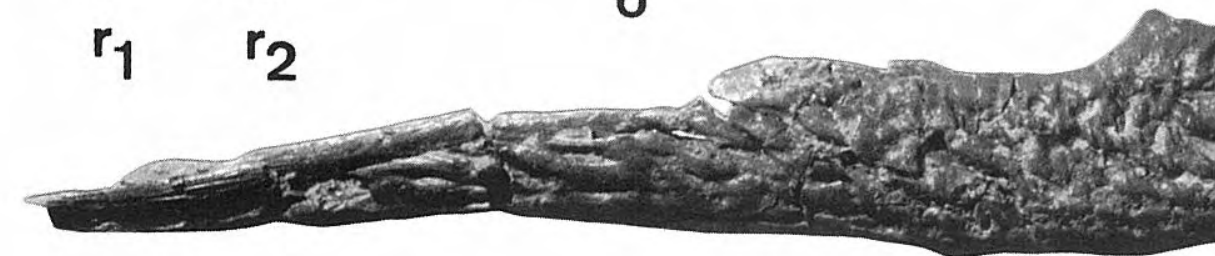

$q_{1}$

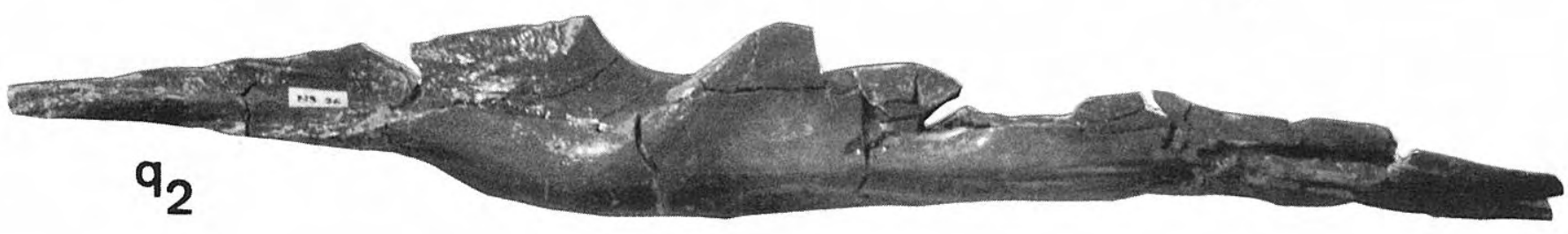


Pterigoides (Fig. 3d). Sólo se conservan las extremidades posteriores de los pterigoides izquierdo y derecho. Se observa la faceta de articulación para el ectopterigoides. El pterigoides se prolonga una cierta distancia por detrás del ectopterigoides.

Maxilar. Los restos maxilares son muy fragmentarios. Su identificación sólo es posible gracias a la presencia de alveolos dentarios, todos ellos seccionados o incompletos.

Angular. Se conserva un pequeño fragmento de angular derecho. La superficie ventral es ancha, convexa y presenta un estrecho surco longitudinal que separa una región ornamentada (lateral) de una región lisa (ventromedial). Se interpreta que este resto proviene de la rama horizontal del angular, aunque el borde ventral de la fosa mandibular externa no se ha conservado.

Articular (Fig. 3e). Se han hallado las extremidades distales de los dos articulares. El proceso retroarticular es subtriangular a redondeado en vista dorsal. La superficie articular es lisa y ligeramente cóncava. Se observa una superficie lateral de sutura para el suprangular y el angular. El foramen aerum no está conservado y se desconoce cuál era su posición en el proceso retroarticular.

\section{- Restos craneales aislados (Figs. 3f-j, 4c)}

Otros restos craneales aislados consisten en fragmentos de maxilar, frontal, postorbital, escamoso, yugal y exoccipital. Estos restos provienen de diferentes yacimientos y pertenecen a distintos individuos.

Maxilar. Hay varios fragmentos maxilares. El más completo contiene 4 alveolos con restos dentarios mal conservados (N3-12). El diámetro máximo de los alveolos es de $6 \mathrm{~mm}$. En otro fragmento de maxilar (BT2-14), el diámetro de los alveolos es de $5 \mathrm{~mm}$.

Frontal (Fig. 3f). El espécimen N2-26 es muy fragmentario y pertenece a un individuo más pequeño que BF2-2. La rotura anterior se sitúa a la altura de la superficie de sutura para el prefrontal. La rotura posterior no permite observar la sutura frontoparietal. En comparación con BF2-2, el espacio interorbital es cóncavo y los bordes de la órbita están más marcados. Esta morfología parece ser variable en Diplocynodon y otros cocodrilos.

Postorbital (Fig. 3g). N2-11 es un postorbital izquierdo de tamaño relativamente grande. El hueso está erosionado y no pueden reconocerse las superficies de sutura para el frontal y parietal (anterior) y para el escamoso (posterior). Sólo se conserva parte del borde anterolateral de la fosa supratemporal. Toda la superficie dorsal está ornamentada con grandes alveolos. El pilar postorbital está roto y, en su parte dorsal, presenta tres pequeños forámenes. Estos se disponen sobre una superficie plana o ligeramente cóncava, a diferencia de los géneros de cocodrilos actuales Alligator y Caiman, que poseen una cresta marcada.

Escamoso (Figs. 3h-i). Hay dos fragmentos de escamosos izquierdos, pertenecientes a dos individuos de diferente tamaño. En ambos casos, se trata del borde posterolateral de la "tabla craneal". En vista occipital, el borde posterior es liso, mientras que, en vista lateral, se observa un reborde ornamentado. En vista dorsal, N1-62 presenta una ornamentación poco marcada formada por orificios subcirculares que se extienden hasta el proceso posterior del escamoso, mientras que N5-12 sigue un patrón distinto, con orificios más profundos, ovalados o acanalados, y un proceso posterior liso. El ejemplar N1-62 pertenecería a un individuo inmaduro y N5-12 a un individuo adulto.

Yugal. CJ-7 es un fragmento de yugal derecho erosionado. La superficie externa es convexa y muestra una ornamentación heterogénea. La superficie interna es lisa y se reconoce la región basal del proceso ascendente de la barra postorbital. La barra infratemporal no se ha conservado. El carácter fragmentario del espécimen no permite conocer el tamaño del foramen sifonial.

Exoccipital (Fig. 3j, 4c). Un exoccipital derecho incompleto (N1-7) es el único resto hallado del basicráneo. Se trata de un hueso cuyo aspecto externo es liso y que conserva la superficie de sutura para el basioccipital y parte de las superficies de sutura para el supraoccipital, el cuadrado y, probablemente, el opistótico, así como el borde lateral del foramen magno. Se observan dos orificios externos e internos para el nervio XII y un orificio de mayor tamaño para el foramen vago, por el que pasan los nervios IX-X-XI y la vena yugular interna. Este último foramen presenta dorsalmente una cresta ósea. El exoccipital está roto lateralmente en la región del proceso paraoccipital, aunque se conserva parte del foramen del canal cranio-cuadrado. El proceso exoccipital está roto a la altura del orificio posterior para la arteria carótida interna. N1-7 pertenece probablemente a un individuo inmaduro.

- Restos mandibulares aislados (Figs. 3k-q, 4d-f)

Hay cuatro dentarios fragmentarios, un angular, un suprangular y un articular. Los restos provienen de yacimientos e individuos diferentes. En función del tamaño relativo, se considera que están representados varios estadios de crecimiento. El pequeño tamaño de los dentarios sugiere que pertenecen a individuos inmaduros, con una longitud estimada de la mandíbula igual o inferior a $20 \mathrm{~cm}$. Por otra parte, un fragmento de suprangular de gran tamaño es equivalente al del espécimen MNHN SG 541 de Diplocynodon rateli y pertenece probablemente a un individuo adulto cuyo cráneo medía unos $40 \mathrm{~cm}$ la longitud. Este tamaño indica un cocodrilo de unos $3 \mathrm{~m}$ de longitud total.

Dentario (Figs. 3k-n, 4d-e). N2-30 es un fragmento de dentario izquierdo que contiene los nueve primeros alveolos y parte de la sínfisis mandibular. Se observan restos de dientes en los alveolos 1 (seccionado), 2, 3, 6, 7 y 8, pero únicamente el tercer alveolo conserva parte de la corona de un diente. BF-38 y BF-46 son dos fragmentos de dentarios derechos que conservan la sínfisis. BF-36 contiene los alveolos 2 a 8 y parte de los alveolos 1 y 9 , ambos seccionados. Los alveolos 2, 3, 4 y 5 poseen restos dentarios 


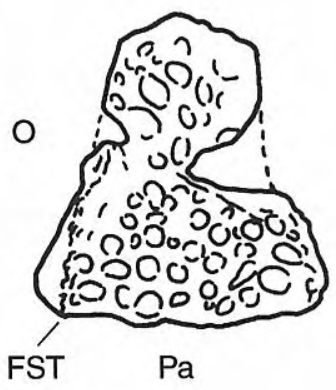

a

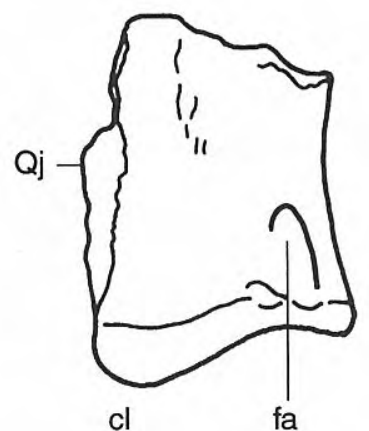

$\mathrm{cl}$

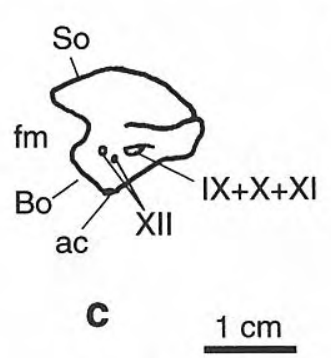

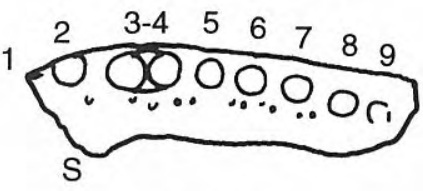

d

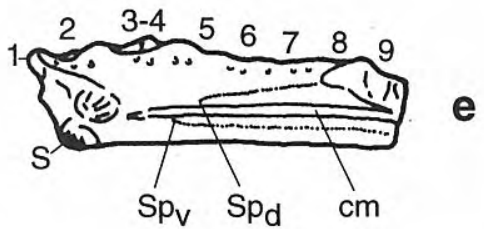

b

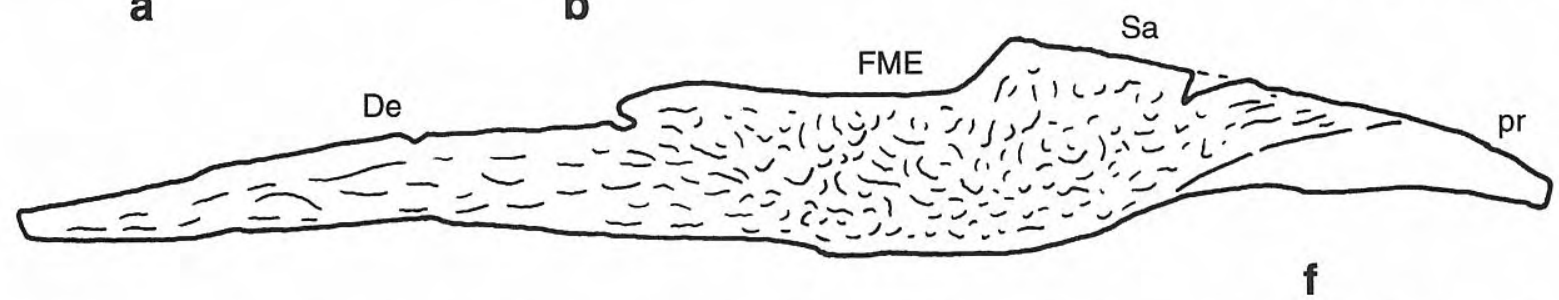

Figura 4. Dibujo esquemático de algunos elementos craneales y mandibulares de Diplocynodon sp. de las Bardenas Reales de Navarra. a, BF2-2, frontal en vista dorsal; b, BF2-2, cuadrado izquierdo en vista dorsal; c, N1-7, exoccipital en vista posterior; d-e, BF-38, dentario derecho en vistas dorsal y medial; f, angular izquierdo en vista lateral. Leyenda (véase también la Fig. 2): ac, arteria carótida; cl, cóndilo lateral; cm, canal de Meckel; fa, foramen aerum; fm, foramen magno; pr, proceso retroarticular; s, sínfisis mandibular; $\mathrm{Sp}_{\mathrm{d}}$, impronta de la rama dorsal del esplenial; $\mathrm{Sp}_{\mathrm{V}}$, impronta de la rama ventral del esplenial; 1-9, alveolos; XII, nervio hipogloso; IX+X+XI, foramen vago.

Schematic drawing of some skull and mandibular remains of Diplocynodon sp. from the Bardenas Reales of Navarre. $\boldsymbol{a}, B F 2-2$, frontal in dorsal view; $\boldsymbol{b}, B F 2-2$, left quadrate in dorsal view; $\boldsymbol{c}, N 1-7$, exoccipital in posterior view; d-e, BF-38, right dentary in dorsal and medial views; $f$, left angular in lateral view. Key (see also Fig. 2): ac, carotid artery; cl, lateral condyle; cm, Meckelian canal; fa, foramen aerum; fm, foramen magnum; pr, retroarticular process; $s$, mandibular symphysis; $S p_{d}$, mark of the dorsal ramus of the splenial; $S p_{v}$, mark of the ventral ramus of the splenial; $1-9$, alveoli; XII, hypoglossus nerve; $I X+X+X I$, foramen vagus.

in situ. En BF-46 se conservan los alveolos 2 a 7 , con un diente fragmentario en el alveolo 6. Los alveolos 1 y 8 están seccionados. Por último, BT3-182 es un fragmento de dentario que conserva únicamente 4 alveolos, 3 de ellos con restos dentarios in situ. Se interpreta que el espécimen corresponde a los alveolos 5 a 8 de una rama mandibular izquierda. De todas estas piezas, la de mayor tamaño (N230) mide $5,5 \mathrm{~cm}$ de longitud.

La forma general del dentario es grácil. El tercer y cuarto alveolos son de mayor tamaño que los restantes y contiguos, estando separados por un borde alveolar muy delgado. Estos alveolos albergaban dos dientes caniniformes, como es característico en el género Diplocynodon Pomel, 1847 (Berg, 1966). La sínfisis mandibular es muy corta y estrecha, extendiéndose hasta la altura del tercer diente (Fig. 6). La sínfisis es asimétrica, con una rama dorsal ligeramente más larga que la ventral, como ocurre en los géneros actuales Crocodylus y Caiman. El esplenial no se conserva en ninguna de las mandíbulas. No obstante, su forma y posición pueden reconocerse a partir de las marcas presentes en el dentario. El esplenial no participa en la sínfisis mandibular. La rama ventral del esplenial está más proyectada anteriormente que la rama dorsal y alcanza el nivel del cuarto alveolo (frente al quinto o sexto alveolo en la rama dorsal). En vista lateral, los alveolos 3 y 4 se disponen formando un resalte dorsal, mientras que los alveolos situados posteriormente (hasta el octavo o noveno conservados) dibujan un arco cóncavo. El tipo de ornamentación permite diferenciar dos regiones en vista lateral: una región anterior, situada cerca del borde dorsal, formada por pequeñas depresiones circulares, y otra, posterior y ventral, compuesta por pequeños surcos longitudinales. En vista oclusal, se observa que los alveolos 3 y 4 son claramente más grandes que los demás. El diámetro mesiodistal del cuarto alveolo es comparable al del tercero, pero puede variar según los individuos: mayor en N2-30, similar en BF-38 y menor en BF-46 (Fig. 7). Por su parte, los alveolos 5, 6 y 7 presentan diferencias mínimas de tamaño y son iguales o ligeramente mayores que los alveolos 8 y 9 . El patrón observado en el material de las Bardenas es comparable al conocido en las diversas especies del género Diplocynodon (cf. Berg, 1966; Buscalioni, 1986a).

Angular (Fig. 3q, 4f). Se conserva un angular izquierdo relativamente completo (N5-26). Se trata de un hueso alargado y convexo lateralmente, con una rama ascendente posterior que se articula con el suprangular y el articular y una rama horizontal que forma el borde ventral de la fosa mandibular externa y se articula con el dentario, el esplenial y el coronoides. El proceso ascendente del angular no sobrepasa en altura el de la fenestra mandibular externa. El 
tamaño de esta fosa es relativamente pequeño. En la zona donde el angular se articula con el esplenial existe una fosa intermandibular inferior de pequeño tamaño. La superficie de sutura con el dentario es oblicua, pero este hueso no llega a acuñarse por debajo de la fosa mandibular externa, como ocurre en los géneros de cocodrilos actuales (Caiman, Crocodylus). A diferencia de los caimanes adultos, la superficie de sutura entre el angular y el suprangular no está situada en el margen ventral de la fosa mandibular externa sino localizada en el borde posterodorsal de la misma. En cualquier caso, la sutura angular-suprangular está situada en posición ventral con respecto a la observada en Alligator mississippiensis. La ornamentación lateral del angular es heterogénea.

Suprangular (Figs. 3o-p). Hay dos fragmentos de suprangulares derechos, uno de gran tamaño (BT3-183) y otro de tamaño ligeramente inferior (N2-18). Ambos fragmentos corresponden a la región de la mandíbula situada por detrás de la fosa mandibular externa. El borde posterodorsal de esta fosa está parcialmente conservado en el ejemplar N2-18. El espécimen BT3-183 presenta medialmente una amplia superficie de sutura para el articular $y$, ventralmente, parte de la superficie de sutura para el angular. A diferencia de los cocodrilos actuales, el suprangular de las Bardenas es relativamente alto, ancho y está profusamente ornamentando en toda su superficie lateral.

Articular. El ejemplar RB1-4 es un fragmento de articular derecho correspondiente a la extremidad distal del proceso retroarticular. Su forma es comparable a la de BF2-2 pero su tamaño es considerablemente menor.

\footnotetext{
- Dientes (Fig. 3r)

Todos los yacimientos de las Bardenas han
}

proporcionado restos dentarios. Los dientes de tamaño centimétrico han sido recogidos en superficie, mientras que los de tamaño milimétrico se han obtenido mediante el lavado y tamizado del sedimento. La mayoría de los dientes conserva únicamente la corona. En aquellas piezas que conservan la raíz, existe una pequeña constricción entre ésta y la base de la corona. El diente de mayor tamaño (CJ-9) conserva la corona y parte de la raíz: la corona mide $15 \mathrm{~mm}$ de longitud y $8 \mathrm{~mm}$ de diámetro mesiodistal. Las dimensiones de este diente son similares a las de los dientes, muy fragmentarios, del individuo BT2-2. Los dientes más pequeños miden menos de $2 \mathrm{~mm}$ de longitud.

Los dientes mejor conservados (BF-47, BF-49) son cónicos y presentan un esmalte con una ornamentación casi lisa, formada por crestas muy finas subparalelas que se extienden desde la base de la corona hasta el ápice. La región apical presenta generalmente una ornamentación filosa o anastomosada más marcada. Las carenas mesial y distal están bien definidas y son lisas. La base de la corona es de sección circular o ligeramente oval.

\section{Esqueleto axial}

Consiste en restos vertebrales de las regiones cervical, dorsal y caudal. Todas las vértebras están desarticuladas y pertenecen a individuos diferentes. El diferente tamaño de las mismas sugiere que están representados varios estadios ontogéneticos, incluyendo formas juveniles (con el arco neural no fusionado al centro) y formas que, sin haber alcanzado un crecimiento completo, presentan un grado de desarrollo más avanzado (con el arco neural unido al centro, aunque se observa la sutura entre ambos). Brochu (1996) considera que el cierre de la sutura centro-neural de las vértebras cervicales es un buen indicador del grado de madurez de un cocodrilo eusuquio, aunque ello no significa necesariamente que el individuo haya alcanzado su máximo tamaño corporal.

Figura 5. Restos postcraneales de Diplocynodon sp., Mioceno Inferior de las Bardenas Reales de Navarra; a-h, Restos vertebrales: a, BF2-2, proceso odontoides del axis en vista anterior (a1) y dorsal (a2); b, BT3-69, primera vértebra postaxial en vista ventral (b1) y dorsal (b2); c, N4-13, cervical posterior en vista ventral; d, BF-48, centro vertebral de la transición cervicotorácica en vista ventral (d1) y dorsal (d2); e, BT3-177, vértebra dorsal en vista lateral (e1) y anterior (e2); f, BT3-181, centro dorsal en vista lateral; g, BF-31, vértebra caudal anterior en vista lateral; h, BF-75, vértebra caudal anterior en vista lateral; i-k, Restos apendiculares: i, BF-51, fémur izquierdo en vista dorsomedial (i1), posterolateral (i2) y anteromedial (i3); j, BF-32, fémur izquierdo en vista anteromedial; k, BT3-8, tibia derecha en vista dorsal (k1) y posterolateral (k2); l-p, Osteodermos: 1, BF-163, osteodermo juvenil en vista dorsal; m, BF50, osteodermo dorsal; $\mathrm{n}, \mathrm{CV}-21$, osteodermo ventral (fragmento de la pieza anterior); o, BF-162, osteodermo ventral (fragmento de la pieza anterior); p, BF-45, osteodermo ventral (pieza posterior) en vista dorsal. Escala: 2 $\mathrm{cm}$. Todas las figuras a tamaño natural.

Postcranial remains of Diplocynodon sp., Early Miocene of the Bardenas Reales of Navarre; $\boldsymbol{a}$ - $\boldsymbol{h}$, Vertebral remains: a, BF2-2, axial odontoid process in anterior (a1) and dorsal (a2) views; $b$, BT3-69, first postaxial vertebra in ventral (b1) and dorsal (b2) views; $c, N 4-13$, posterior cervical in ventral view; $d, B F-48$, centrum from the cervical-dorsal transition in ventral (d1) and dorsal (d2) views; e, BT3-177, dorsal vertebra in lateral (e1) and anterior (e2) views; $f$, BT3-181, dorsal centrum in lateral view; $g, B F-31$, anterior caudal vertebra in lateral view; $h, B F-75$, anterior caudal vertebra in lateral view; $i-k$, Appendicular remains: $i, B F-51$, left femur in dorsomedial (i1), posterolateral (i2) and anteromedial (i3) views; $j, B F-32$, left femur in anteromedial view; $k$, BT3-8, right tibia in dorsal ( $k 1)$ and posterolateral ( $k 2$ ) views; l-p, Dermal scutes: l, BF-163, juvenile scute in dorsal view; $m, B F-50$, dorsal scute; $n, C V-21$, ventral scute (fragment of the anterior ossification); $o, B F-162$, ventral scute (fragment of the anterior ossification); $p, B F-45$, ventral scute (posterior ossification). Scale: $2 \mathrm{~cm}$. All photographs at natural size. 
No se conoce con exactitud el número de vértebras que componían la columna vertebral de Diplocynodon. Un representante actual de los aligatóridos, como Alligator mississippiensis, posee 24 vértebras presacras ( 9 cervicales sin contar el proatlas- y 15 dorsales), 2 sacras y entre 30 y 40 caudales (Brochu, 1996). Todas las vértebras de los yacimientos de las Bardenas son procélicas, como es típico de los cocodrilos eusuquios, aunque éste carácter también se conoce en formas no eusuquias (Buscalioni y Sanz, 1990; Michard et al., 1990).

Vértebras cervicales (Figs. 5a-c). Se hallaron varias vértebras cervicales y todas ellas conservan únicamente el centro vertebral con la superficie de sutura para el arco neural. Los centros son de pequeño tamaño y no suelen sobrepasar los $2 \mathrm{~cm}$ de longitud.

El único hueso conservado del complejo atlas-axis es un proceso odontoides libre, no fusionado al axis. Pertenece al mismo individuo que ha proporcionado restos craneales y mandibulares (BF2-2). Es cruciforme, con una base ancha, dos apófisis laterales y una superficie dorsal plana. El odontoides (originalmente el centro del atlas) se fusiona tempranamente al axis en Crocodylus porosus y más tardíamente en Alligator mississippiensis (cf. Brochu, 1996).

El ejemplar BT3-69 es un centro cervical anterior. Carece de hipapófisis y las parapófisis, bien desarrolladas,
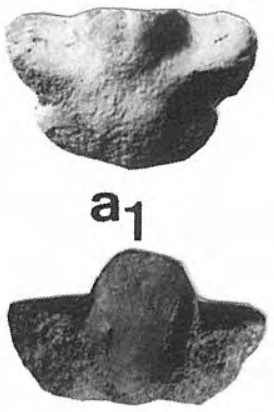

$a_{2}$

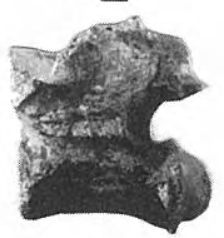

$\mathrm{e}_{1}$

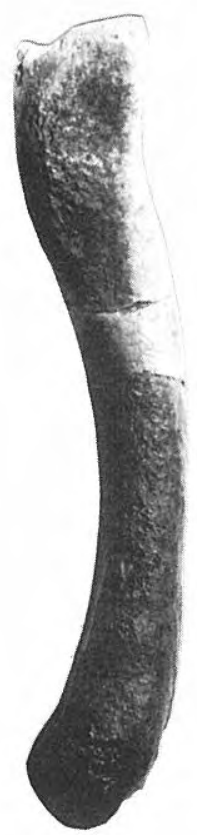

$i_{1}$

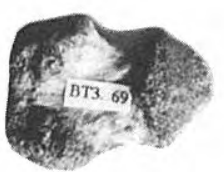

$b_{1}$

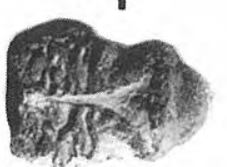

$b_{2}$

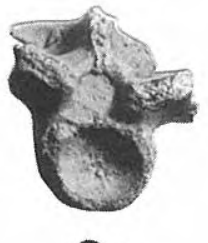

$e_{2}$

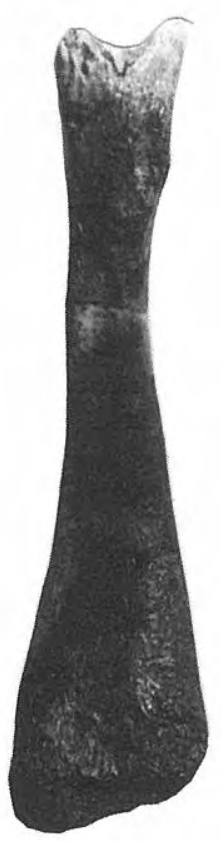

$i_{2}$

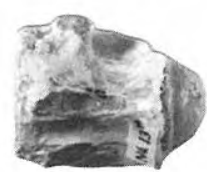

C

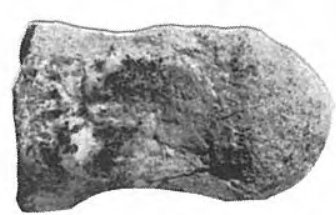

f
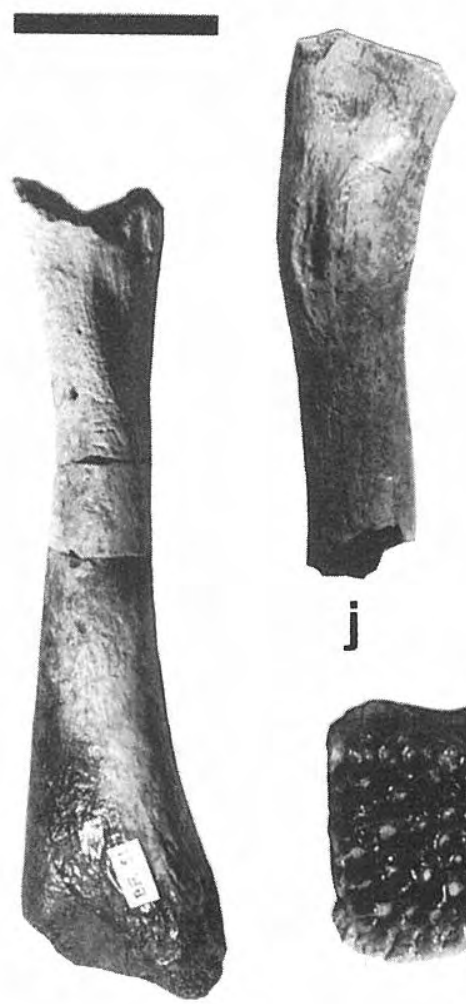

$\mathrm{i}_{3}$

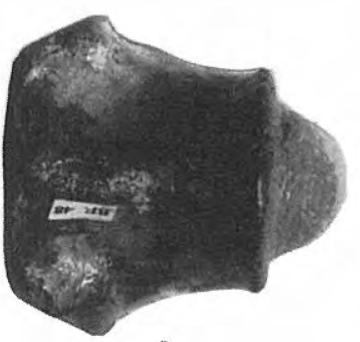

$d_{1}$

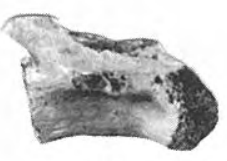

g
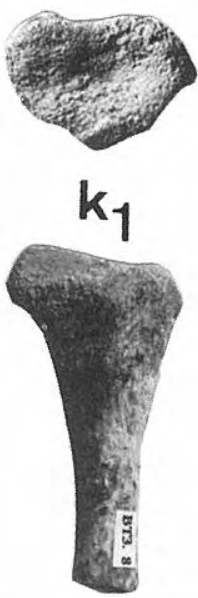

$k_{2}$

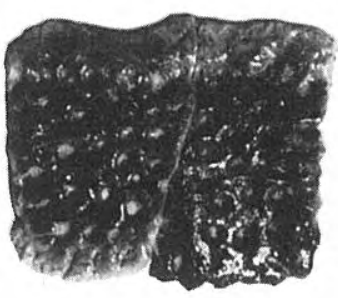

$m$
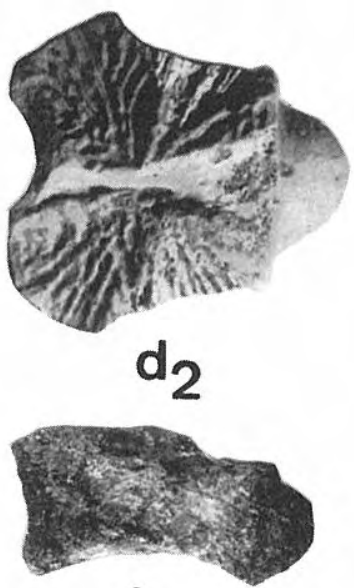

h
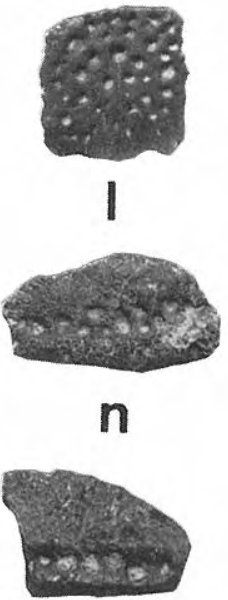

o

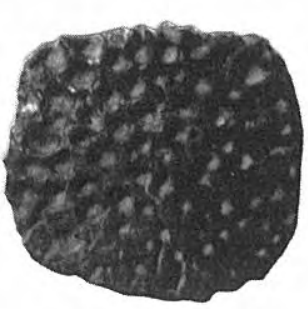

p 
están situadas en posición ventral. Estas características sugieren que se trata de la primera cervical postaxial. En otros especímenes, como N3-9 y N4-13, la presencia de hipapófisis y el hecho de que las parapófisis se sitúen en la parte media del centro en vista lateral indican que ocupaban una posición posterior en la serie cervical. La hipapófisis del espécimen N3-9 está situada en la parte anterior del centro, mientras que en el N4-13 se extiende ventralmente hasta el tercio distal del centro.

Vértebras de la transición cervicotorácica (Fig. 5d). BF48 y N1-18 son dos vértebras de la transición cervicotorácica. BF-48 es de mayor tamaño pero conserva únicamente el centro (longitud 3,3 cm). N1-18 presenta la base del arco neural fusionada al centro $(2,1 \mathrm{~cm}$ de longitud). En ambos casos, las parapófisis están menos proyectadas que en las vértebras cervicales descritas anteriormente y se sitúan en la región de la sutura centroneural. Las hipapófisis están bien marcadas ventralmente. Estos procesos arrancan ligeramente por detrás del borde anteroventral del cotilo y se prolongan ventralmente hasta más allá de la mitad de la longitud del centro.

Vértebras dorsales (Figs. 5e-f). BF-17 es una vértebra dorsal anterior de pequeño tamaño. Conserva parte del centro y del arco neural fusionado a éste. El canal neural es relativamente grande. El centro conserva una hipapófisis ventral. En BF-18 el centro (longitud de 1,5 cm) y el arco neural no están totalmente fusionados sino separados por una sutura abierta, lo que sugiere que la vértebra pertenecía a un individuo juvenil. El cóndilo y el cotilo son más anchos que altos y la superficie ventral del centro (longitud aproximada $2 \mathrm{~cm}$ ) es redondeada y carece de hipapófisis. Esto indica que la vértebra pertenece a la parte media o posterior de la serie dorsal. Las mismas características se observan en otros ejemplares (BT3-76, BT3-177, BT3-178). BT3-68, ВT3-179, ВT3-180 у BT3-181 presentan una morfología similar pero son vértebras de mayor tamaño (longitud máxima del centro de $3,2 \mathrm{~cm}$ ). Estas vértebras ofrecen claras muestras de meteorización.

Vértebras caudales (Fig. $5 \mathrm{~g}$-h). Hay varias vértebras caudales anteriores (BF-31, BF-75), cuyo centro (longitud 2 $\mathrm{cm})$ es dos veces más largo que alto o ancho y posee un surco ventral dispuesto según el eje longitudinal. Estas vértebras presentan superficies de articulación para los arcos hemales, así como procesos transversos. El centro de N5-7 es muy alargado (longitud $3 \mathrm{~cm}$ ), comprimido lateralmente y su superficie ventral es muy estrecha. Esta vértebra conserva los procesos transversos y, aunque es posterior en la serie a las descritas anteriormente, pertenece a la mitad proximal de la región caudal.

\section{Esqueleto apendicular}

Está representado principalmente por cinco huesos del miembro posterior: tres fémures izquierdos (BF-32, BF-51, BT3-62), una tibia izquierda (BF-99) y una tibia derecha (BT3-8), todos ellos incompletos. El grado de robustez de los huesos es similar al de otros aligatoroideos, con excepción del género Borealosuchus, que es muy grácil (Brochu, 1997).
Fémur (Figs. 5i-j). Uno de los fémures (BF-32) conserva la mitad proximal, pero la cabeza articular está rota. El segundo fémur (BF-51) conserva dos tercios del hueso, incluída la extremidad distal; su longitud es de $8,5 \mathrm{~cm}$. El tercer ejemplar (BT3-62) es una extremidad distal. Se estima que la longitud total del fémur mediría $10-12 \mathrm{~cm}$. La diáfisis presenta una fuerte torsión y es sigmoidal en vista lateral. Los cóndilos distales están erosionados, aunque se observa que el cóndilo lateral es más ancho y está distalmente más proyectado que el medial. El cuarto trocánter es poco prominente. En posición medial al cuarto trocánter existe una depresión poco profunda para la inserción muscular $(M$. caudi-femoralis longus).

Tibia (Fig. 5k). Los dos fragmentos tibiales pertenecen a individuos de distinto tamaño. La tibia más pequeña conserva únicamente la extremidad proximal, la más grande posee además parte de la diáfisis. La articulación proximal es oval. Los cóndilos externo e interno son redondeados y están bien definidos, mientras que la cresta cnemial está poco marcada. La diáfisis es ligeramente curva y presenta una sección oval.

\section{Esqueleto dérmico}

Hay numerosos restos del dermatoesqueleto, aunque sólo unos pocos están completos (Figs. 5l-p). Los osteodermos dorsales son subrectangulares (longitud $2,8 \mathrm{~cm}$ y anchura 3,2 $\mathrm{cm}$ en el ejemplar BF-50) y poseen una quilla dorsal poco prominente. La superficie dorsal está ornamentada, con excepción de una estrecha banda lisa en posición anterior, que es la superficie de solapamiento con la que se articula el osteodermo situado en posición anterior. Los bordes medial y lateral del ejemplar BF-50 tienen superficies de sutura. La quilla media tiene una disposición ligeramente lateral y es oblicua. Algunos de los osteodermos de pequeño tamaño (longitud y anchura de 1,3 cm) muestran una ornamentación poco desarrollada. Estos osteodermos pertenecen probablemente a formas juveniles. Los osteodermos ventrales carecen de quilla y están formados por dos piezas, una posterior completamente ornamentada y una anterior más estrecha que posee una banda lisa y una región ornamentada (Kälin, 1955: fig. 66; Laemmert, 1993). El ejemplar BF-45 es la pieza posterior de uno de estos osteodermos compuestos y el CV-21 es parte de la pieza anterior de otro osteodermo, como sugiere la presencia de una superficie de sutura en los bordes anterior y posterior, respectivamente. Todos los osteodermos hallados son elementos aislados y no ofrecen información sobre la composición y disposición del dermatoesqueleto.

\section{DISCUSIÓN}

\section{ATRIBUCIÓN DEL MATERIAL DE LAS BARDENAS A DIPLOCYNODON}

El cocodrilo de las Bardenas comparte al menos una sinapomorfía con los Eusuchia: la presencia de vértebras procélicas. Algunas formas no eusuquias también presentan este carácter, pero es debido a un fenómeno de 
paralelismo (Benton y Clark, 1988; Michard et al., 1990; Clark, 1994). La existencia de hipapófisis prominentes en los centros cervicales es típica de los eusuquios (poco prominentes en Gavialis), pero también se da en dirosáuridos y Bernissartia, aunque en este último están poco marcadas (Norell y Clark, 1990).

El cocodrilo de las Bardenas comparte con los Alligatoroidea la presencia de un foramen aerum en posición dorsal del cuadrado (Brochu, 1999). Además, comparte con los Diplocynodontinae de Brochu (1999), clado compuesto por Diplocynodon y Baryphracta, la presencia de osteodermos ventrales formados por osificaciones pares (Buscalioni et al., 1992). Según Brochu (1997, 1999), la existencia de osteodermos ventrales pares en dos especies del eusuquio primitivo Borealosuchus (B. formidabilis, B. wilsoni) puede ser debida a convergencia. Los osteodermos bipartitos de algunos aligatóridos modernos (Buscalioni et al., 1992; Brochu, 1999) no son homólogos, ya que no hay un recubrimiento anteroposterior como en Diplocynodon.

El cocodrilo de las Bardenas y las siguientes especies de Diplocynodon: $D$. rateli, $D$. tormis, $D$. darwini, $D$. hantoniensis y $D$. styriacus, así como la forma asignada a Diplocynodon sp. de Mancy, comparten la exclusión del esplenial de la sínfisis mandibular (Buscalioni et al., 1992; Brochu, 1999). La participación del esplenial en la sínfisis mandibular parece ser la condición primitiva en los Alligatoroidea (según Brochu, 1999). El material inédito del Paleoceno de Cernay-Mont-Berru, asignado provisionalmente en este trabajo a aff. Diplocynodon sp., comparte también la condición primitiva, por lo que no se descarta que la exclusión del esplenial de la sínfisis mandibular haya podido adquirirse en varias ocasiones entre los Alligatoroidea, tanto en Alligatoridae como en Diplocynodontinae.

Otro carácter compartido por el cocodrilo de las Bardenas y Diplocynodon es la presencia de alveolos dentarios tercero y cuarto del mismo tamaño y confluentes. Este carácter, utilizado por Rauhe y Rossman (1995) para definir el clado Leidyosuchidae, es considerado plesiomórfico por Buscalioni et al. (1992). Brochu (1999) interpreta que se trata de una simplesiomorfía o de una sinapomorfía ambigua de Diplocynodon, lo que implica una transformación autapomórfica idéntica en Leidyosuchus canadensis. Además de las especies de Diplocynodon y Leidyosuchus canadiensis, el doble diente caniniforme está presente en aff. Diplocynodon sp. de Cernay-Mont-Berru (Champaña), Bernissartia y Borealosuchus (Brochu, 1999: fig. 34). En los Globidonta de Brochu (1999), es decir todos los aligatoroideos no "diplocinodontinos", el cuarto alveolo del dentario es mayor que el tercero y están separados uno de otro. Frey et al. (1987) sugieren que los alveolos tercero y cuatro de Baryphracta están separados pero, como todos los especímenes conocidos presentan las mandíbulas cerradas, no es posible confirmarlo (Brochu, 1999).

Los dentarios de los yacimientos de las Bardenas se diferencian de las especies conocidas de Diplocynodon en la menor longitud de la sínfisis mandibular, que se prolonga únicamente hasta el tercer alveolo. Sólo los ejemplares de Diplocynodon sp. de Mancy presentan una sínfisis tan corta, aunque ésta puede alcanzar en algún dentario el cuarto alveolo. Teniendo en cuenta la variabilidad morfológica observada en las sínfisis mandibulares de los aligatoroideos y a la espera de una revisión del material europeo del género Diplocynodon y formas afines, asignamos el material de las Bardenas a Diplocynodon sp. indet.

\section{ANÁLISIS DE LA VARIABILIDAD MORFOLÓGICA}

Como ya se ha comentado previamente, la variabilidad morfológica observada en los restos de cocodrilo de las Bardenas es muy pequeña, por lo que todo el material estudiado se asigna a la misma especie. Las piezas halladas de un mismo elemento cranial o mandibular (por ejemplo, frontal, escamoso, dentario, suprangular, dientes) y postcraneal (vértebras, fémures, tibias) no presentan rasgos diferentes como para justificar la presencia de dos o más géneros. Al contrario, las diferencias observadas son poco significativas desde un punto de vista sistemático. Algunas de ellas (grado de ornamentación del escamoso o de los osteodermos) pueden ser de carácter ontogenético. Otras diferencias (frontal con un espacio interorbital plano o cóncavo y bordes de la órbita más o menos marcados) se interpretan como debidas a una variación intraespecífica, ya que se pueden observar en el seno de especies actuales de cocodrilos. Por lo que respecta a los dientes, su variabilidad morfológica entra dentro de la observada para el género Diplocynodon de los yacimientos paleógenos de la Cuenca del Duero (véase Martín de Jesús et al., 1987).

La morfología craneal de los restos fósiles de los yacimientos de las Bardenas no difiere de la característica de la especie Diplocynodon rateli (neotipo y otro material conservado en el MNHN de París). No obstante, la sutura frontoparietal del cocodrilo de las Bardenas es relativamente recta, mientras que, según Brochu (1999: fig. 40A), esta sutura es curva en algunos especímenes de D. rateli (MNHN SG 557). Este carácter parece presentar una gran variabilidad individual, ya que otros ejemplares asignados a $D$. rateli poseen una sutura frontoparietal más o menos rectilínea (Brinkmann y Rauhe, 1998). Así mismo, la sutura frontoparietal tiende a ser recta en $D$. tormis (Buscalioni et al., 1992: figs. 2, 4), D. hantoniensis (Vignaud et al., 1996: fig. a) y D. darwini (Brochu, 1999: fig. 11c). Brochu (1999) considera que la presencia de una sutura frontoparietal más o menos rectilínea y situada entre las fosas supratemporales es una sinapormorfía del clado Diplocynodontinae (formado por los géneros Diplocynodon y Baryphracta), pero reconoce que este carácter es variable entre los eusuquios.

La posición de la sutura frontoparietal con respecto a las fosas supratemporales varía según los Diplocynodontinae de Brochu (1999). En Diplocynodon, al menos en las especies $D$. rateli, $D$. tormis y $D$. styriacus, así como en el material de las Bardenas, el parietal se extiende hasta el borde anterior de las fosas 


\begin{tabular}{|c|c|c|c|c|c|c|c|}
\hline $\begin{array}{c}\text { Taxón } \\
\text { (localidad y } \\
\text { edad relativa) }\end{array}$ & $\begin{array}{l}\text { Número de } \\
\text { ejemplares } \\
\text { (muestra } \\
\text { estudiada) }\end{array}$ & $\begin{array}{l}\text { Extensión de la } \\
\text { sínfisis mandibular } \\
\text { (intervalo de } \\
\text { extensión) }\end{array}$ & $\begin{array}{l}\text { Gran diferencia de } \\
\text { tamaño entre el } 5^{\circ} \\
\text { diente y el doble } \\
\text { caniniforme }\end{array}$ & $\begin{array}{l}\text { Heterodoncia } \\
\text { decreciente } \\
\text { entre los } \\
\text { alveolos } 5 \text { a } 9\end{array}$ & $\begin{array}{c}\text { Espaciado } \\
\text { interalveolar } \\
\text { entre los } \\
\text { alveolos } 5 \text { a } 9\end{array}$ & $\begin{array}{l}\text { Participación } \\
\text { del esplenial } \\
\text { en la sínfisis } \\
\text { mandibular }\end{array}$ & $\begin{array}{l}\text { Proyección de la } \\
\text { rama ventral del } \\
\text { esplenial con } \\
\text { respecto a la sínfisis }\end{array}$ \\
\hline $\begin{array}{l}\text { Diplocynodon styriacus } \\
\quad \text { (Artenay, MN 4a) }\end{array}$ & 6 & $\begin{array}{l}4^{\circ} \text { alveolo (del } \\
\text { inicio al final) }\end{array}$ & $\begin{array}{c}\text { En la mitad de los } \\
\text { ejemplares }\end{array}$ & Regular & Irregular & No & $\begin{array}{c}4^{\circ} \text { alveolo (mitad o } \\
\text { final), muy cerca } \\
\text { de la sínfisis }\end{array}$ \\
\hline $\begin{array}{l}\text { Diplocynodon rateli } \\
\text { (St. Gérand-le-Puy, MN 2a) }\end{array}$ & 10 & $\begin{array}{l}\text { Del } 4^{\circ}(\mathrm{mitad}) \text { al } 5^{\circ} \\
\text { alveolo (inicio) }\end{array}$ & $\begin{array}{l}\text { En todos los } \\
\text { ejemplares }\end{array}$ & Irregular & Irregular & No & $\begin{array}{l}4^{\circ} \text { alveolo (mitad), } \\
\text { cerca de la sínfisis }\end{array}$ \\
\hline $\begin{array}{l}\text { Diplocynodon tormis } \\
\text { (Salamanca, MP 16-17) }\end{array}$ & 1 & $4^{\circ}$ alveolo? & $?$ & $?$ & Irregular & No & $?$ \\
\hline $\begin{array}{l}\text { Diplocynodon sp. } \\
\text { (Mancy, MP 10) }\end{array}$ & 4 & $\begin{array}{l}\operatorname{Del~} 3^{\circ} \text { (final) al } 4^{\circ} \\
\text { alveolo (mitad) }\end{array}$ & En 3 de 4 ejemplares & Regular & Irregular & No & $\begin{array}{c}4^{\circ} \text { alveolo (mitad o } \\
\text { final), alejada de } \\
\text { la sínfisis }\end{array}$ \\
\hline $\begin{array}{c}\text { aff. Diplocynodon sp. } \\
\text { (Cernay-Mont-Berru, MP 6) }\end{array}$ & 5 & $\begin{array}{l}\text { Del } 4^{\circ} \text { (final) al } 5^{\circ} \\
\text { alveolo (final) }\end{array}$ & $\begin{array}{l}\text { En todos los } \\
\text { ejemplares }\end{array}$ & Regular & $\begin{array}{l}\text { Relativamente } \\
\text { regular }\end{array}$ & Sí & $\begin{array}{l}\text { Forma parte de la } \\
\text { sínfisis }\end{array}$ \\
\hline $\begin{array}{l}\text { Diplocynodon sp. } \\
\text { (Bardenas, MN 2b-3) }\end{array}$ & 3 & $\begin{array}{l}3^{\circ} \text { alveolo (del } \\
\text { inicio al final) }\end{array}$ & En 1 de 3 ejemplares & Regular & $\begin{array}{c}\text { Irregular } \\
(2 \text { ejemplares })\end{array}$ & No & $\begin{array}{l}4^{\circ} \text { alveolo (final), } \\
\text { alejada de la sínfisis }\end{array}$ \\
\hline
\end{tabular}

Figura 6. Cuadro resumen de la variabilidad morfológica observada en los dentarios de las Bardenas y material mandibular de comparación perteneciente a Diplocynodon (véase discusión en el texto).

Table showing the morphological variation observed in the dentaries of the Bardenas and other mandibular remains of Diplocynodon (see discussion in the text).

supratemporales, de forma que hay un contacto mínimo entre el parietal y el postorbital. Por el contrario, en el ejemplar asignado a aff. Diplocynodon sp. del Paleoceno de Cernay-Mont-Berru (Champaña), la sutura frontoparietal sólo alcanza la mitad de la longitud de las fosas supratemporales y no hay contacto entre el parietal y el postorbital (condición primitiva). En D. darwini el frontal no participa apenas en la fosa supratemporal, pero existe una variabilidad de carácter ontogenético e intraespecífico (Buscalioni et al., 1992).

La comparación de los dentarios de los yacimientos de las Bardenas con material mandibular del género Diplocynodon y formas afines (Baryphracta, aff. Diplocynodon sp. de Cernay-Mont-Berru, Francia) se ha realizado tomando como referencia los siguientes parámetros:

1) la extensión de la sínfisis mandibular;

2) el tamaño del quinto diente con respecto al doble diente caniniforme;

3) la heterodoncia de los alveolos 5 a 9;

4) el espaciado interalveolar entre los dientes 5 a 9 ;

5) la disposición relativa de las ramas dorsal y ventral del esplenial y su distancia con respecto a la sínfisis mandibular.

Los resultados obtenidos se discuten a continuación (Fig. 6):

1) En Diplocynodon, la extensión de la sínfisis mandibular es variable y se sitúa entre los alveolos 3 y 5 (Brinkmann y Rauhe, 1998). En los ejemplares de Diplocynodon sp. de las Bardenas la sínfisis es muy corta y no se prolonga más allá del tercer alveolo. En
Diplocynodon sp. de Mancy, la sínfisis es corta, alcanzando en unos casos el borde posterior del tercer alveolo y en otros la mitad del cuarto alveolo. En $D$. styriacus de Francia, la sínfisis no se extiende por detrás del cuarto alveolo, mientras que en $D$. rateli y la forma asignada a aff. Diplocynodon sp. de Cernay-Mont-Berru puede alcanzar el quinto alveolo. Buscalioni et al. (1992) indican que la sínfisis de $D$. tormis es corta, pero sin precisar su extensión. En la muestra estudiada, la extensión de la sínfisis mandibular no está directamente correlacionada con el tamaño de la mandíbula (desarrollo ontogenético). No obstante, Brochu (2000) sugiere que podría existir una correlación entre la longitud de la sínfisis y el tamaño corporal en la especie actual Alligator mississippiensis, siendo las mandíbulas de mayor tamaño las que presentan las sínfisis más largas. Esto no es válido para los Crocodylidae, donde la longitud de la sínfisis es específica, aunque varias especies del mismo género pueden tener el mismo tamaño relativo de sínfisis. Brochu (2000) considera que el tamaño de la sínfisis por sí solo no es un criterio suficiente para discriminar entre dos especies diferentes.

2) En la muestra estudiada, todos los dentarios presentan una notable diferencia de tamaño entre el quinto alveolo y el doble caniniforme. En algunos casos, esta diferencia puede ser del orden de un tercio (o incluso la mitad) del diámetro del tercer o cuarto diente. Aunque tal diferencia se observa generalmente en los especímenes de mayor tamaño, también puede apreciarse en mandíbulas de pequeñas dimensiones. En el material de las Bardenas, sólo un dentario de tres presenta el 
quinto alveolo reducido (Fig. 7). En resumen, puede decirse que el tamaño relativo del quinto diente con respecto al doble caniniforme es variable en Diplocynodon, pero siempre claramente inferior. Teniendo en cuenta que la muestra estudiada es muy reducida, los resultados obtenidos son indicativos y no tienen valor estadístico.

3) Los dentarios de las Bardenas presentan una heterodoncia decreciente regular, con tres alveolos 5-6-7 de tamaño inferior al doble caniniforme y dos alveolos 89 de tamaño aún más pequeño (Fig. 7). Este tipo de heterodoncia se observa también en D. styriacus de Artenay y Bézian (Departamento del Gers) (Ginsburg y Bulot, 1997), en Diplocynodon sp. de Mancy y en aff. Diplocynodon sp. de Cernay-Mont-Berru, siendo especialmente remarcable en los ejemplares de Mancy, donde los alveolos son de tamaño muy pequeño. En $D$. rateli, la heterodoncia es irregular: el séptimo alveolo puede ser mayor que el quinto y el sexto, el noveno alveolo mayor o claramente menor que el octavo, etc. Un estudio de este parámetro en otras especies de Diplocynodon es necesario para poder generalizar u obtener conclusiones de orden sistemático.

4) El espaciado interalveolar de la serie mandibular postcaniniforme es variable en Diplocynodon y formas afines. En el material de las Bardenas, el espaciado es regular en un dentario (BF-38) e irregular en los otros dos ejemplares. En aff. Diplocynodon sp. de Cernay-MontBerru el espaciado es relativamente regular, excepto en un dentario (séptimo y octavo alveolos coalescentes). Un espaciado irregular parece ser lo más frecuente en las especies $D$. rateli, D. styriacus y Diplocynodon sp. de Mancy. En D. rateli y D. tormis (A. D. Buscalioni, com. pers.) puede existir un gran espacio entre los alveolos $7 \mathrm{y}$ $8 \mathrm{y}$, en ocasiones, el quinto alveolo es coalescente con el cuarto.

5) En Diplocynodon, el esplenial no participa en la sínfisis mandibular (Buscalioni et al., 1992), a diferencia de Baryphracta del Eoceno de Messel (Frey et al., 1987) y de aff. Diplocynodon sp. de Cernay-Mont-Berru, que conservan la condición primitiva. Las especies de Diplocynodon poseen la rama ventral del esplenial más proyectada en dirección de la sínfisis que la rama dorsal. La distancia entre la extremidad anterior de ambas ramas puede variar: en $D$. rateli y D. styriacus, esta distancia es mayor que en los ejemplares de Diplocynodon sp. de las Bardenas y Mancy. Por otra parte, la rama ventral del esplenial se extiende generalmente hasta la mitad o el final del cuarto alveolo, pero su distancia relativa respecto a la sínfisis varía según las especies: cerca o muy cerca de la sínfisis en $D$. rateli y $D$. styriacus y más alejada en D. hantoniensis (Brochu, 1999: fig. 31B) y las formas de Mancy y las Bardenas.

Los resultados obtenidos son indicativos para los caracteres 1 y 5 . Los caracteres 2,3 y 4 no ofrecen información concluyente.

Por último, la comparación del material postcraneal ofrece escasa información. Un carácter vertebral interesante es la presencia o ausencia de hipapófisis en la primera vértebra postaxial. Como ya se ha comentado

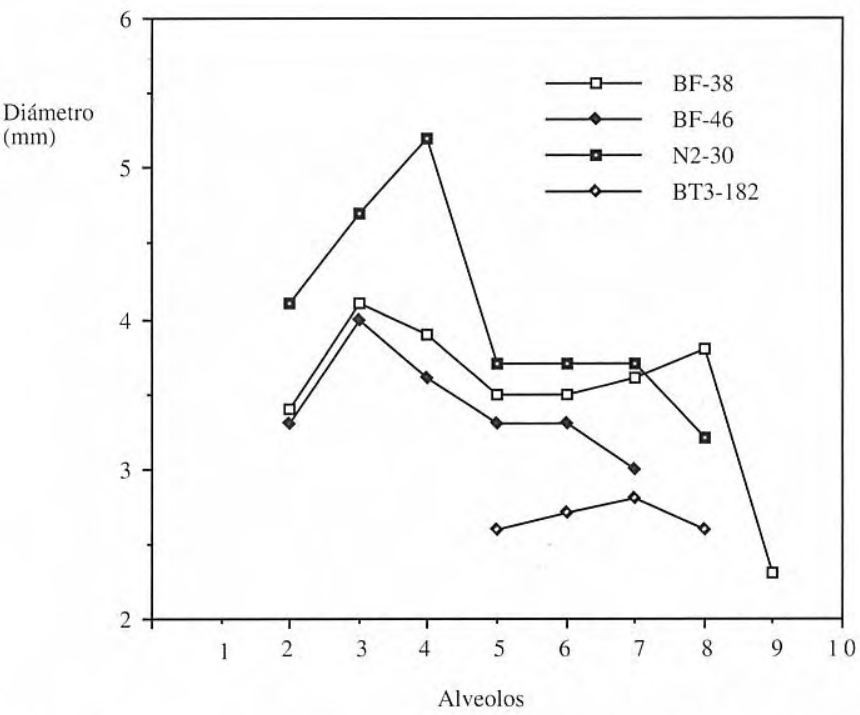

Figura 7. Diámetros mesio-distales (en milímetros) de los alveolos de los restos mandibulares de Diplocynodon sp. de las Bardenas Reales de Navarra.

Mesiodistal diameters (in $\mathrm{mm}$ ) of the alveoli of the mandibles of Diplocynodon sp. from the Bardenas of Navarre.

anteriormente, la forma de las Bardenas presenta una primera vértebra postaxial sin hipapófisis. Brochu (1999: apéndice 2, matriz) considera que la hipapófisis de la tercera cervical es prominente en Diplocynodon hantoniensis y $D$. darwini, pero indica que no se conserva o no puede observarse en $D$. rateli y Barypracta deponiae. Para este autor, todos los aligatoroideos y crocodiloideos poseen hipapófisis en la primera vértebra postaxial, mientras que los eusuquios basales como los géneros Bernissartia y Borealosuchus carecen de ella (Brochu, 1999). No obstante, entre el material de $D$. rateli conservado en París hay varias vértebras cervicales anteriores, pertenecientes a individuos diferentes, que podrían corresponder a la primera postaxial y que carecen de hipapófisis (entre ellas MNHN SG 570, SG 602). Además de D. rateli y de la especie de Diplocynodon de las Bardenas, la primera vértebra postaxial de $D$. styriacus de Artenay (MNHN colección AR), de aff. Diplocynodon sp. de Cernay-Mont-Berru (MNHN BR 3613) y de Stangerochampsa mccabei (Wu et al., 1996), grupo hermano de los Alligatoridae (según Brochu, 1999), está también desprovista de hipapófisis, por lo que la interpretación de este carácter debe revisarse.

\section{RESEÑA HISTÓRICA DEL GÉNERO DIPLOCYNODON}

Pomel (1847) erigió el género Diplocynodon (especie tipo D. rateli Pomel, 1847) a partir de material craneal y postcraneal procedente del Mioceno Inferior (MN2a) de Saint-Gérand-le-Puy (Allier, Auvernia), basándose en la presencia de dos dientes caniniformes contiguos en el tercer y cuarto alveolos de la mandíbula. Desde la época de Pomel se han descrito numerosas especies de 
Diplocynodon en el Paleógeno y Neógeno de Europa (véase Pomel, 1853; Lydekker, 1887; Kuhn, 1936; Berg, 1966; Steel, 1973; Buscalioni et al., 1992). Algunos autores clásicos asignaron originalmente varias de estas nuevas especies a los géneros Alligator y Crocodylus. Aunque se ha descrito el hallazgo de restos de Diplocynodon en Norteamérica, Asia y el norte de Africa, la presencia de este género fuera del continente europeo es dudosa (Buscalioni et al., 1992; Brochu, 1997).

Berg (1966) hizo una revisión completa del material de Diplocynodon. Además de la especie tipo, D. rateli Pomel, 1847 (sinónimo D. gracilis Vaillant, 1872), Berg (1966) incluyó dentro del género las especies A. darwini Ludwig, 1877 y C. ebertsi Ludwig, 1877 del Eoceno Medio de Messel (Hessen) y admitió la validez de las siguientes especies europeas: D. hallensis Kuhn, 1938 del Eoceno Medio de Sajonia, D. hantoniensis (Wood, 1846) del Eoceno Superior de Hampshire, D. gervaisi (Gervais, 1859) del Oligoceno Inferior de Auvernia, D. styriacus (Hofmann, 1885) (sinónimo C. steineri Hofmann, 1885) del Mioceno Inferior de Austria y D. levantinicus Huene, 1963 (in Huene y Nikoloff, 1963) del Mioceno Superior de Bulgaria. Berg (1966) basó principalmente sus diagnosis en el número de dientes premaxilares y maxilares con respecto a los dientes mandibulares y las proporciones relativas entre los alveolos.

Los trabajos de revisión realizados en los últimos años sugieren que las especies $D$. ebertsi y $D$. hallensis son sinónimos de $D$. darwini (véase Grössens-Van Dick, 1979; Buscalioni et al., 1992; Rauhe y Rossman, 1995; Ginsburg y Bulot, 1997). D. gervaisi y D. styriacus pueden ser especies válidas de Diplocynodon (según Buscalioni et al., 1992; Ginsburg y Bulot, 1997) o sinónimos de $D$. rateli (según Rauhe y Rossman, 1995). Por último, la validez de D. levantinicus y $D$. dalpiazi (Fabiani, 1915), especie olvidada por Berg (1966), es dudosa.

Buscalioni et al. (1992) erigieron una nueva especie, D. tormis, del Eoceno Superior de la Cuenca del Duero (Ortega, 1990), y propusieron una nueva definición cladística del género Diplocynodon restringida a tres especies: $D$. rateli, D. tormis y $D$. gervaisi. En este análisis, no se incluyó la especie D. styriacus. Estos autores excluyeron provisonalmente del género las especies $D$. hantoniensis y $D$. darwini. Rauhe y Rossman (1995) y Vignaud et al. (1996) han criticado la exclusión de la especie $D$. hantoniensis del género Diplocynodon. En una revisión preliminar del material europeo, Rauhe y Rossman (1995) consideraron válidas cuatro especies de Diplocynodon: D. darwini, D. tormis, D. hantoniensis y $D$. rateli, siendo $D$. styriacus y $D$. gervaisi sinónimos de $D$. rateli. Los autores alemanes han redefinido el clado Leidyosuchidae, al que asignan los géneros Diplocynodon, Leidyosuchus y Baryphracta. Sin embargo, Brochu (1997) considera que Leidyosuchus es un taxón parafilético y retiene únicamente la especie $L$. canadensis entre los Alligatoroidea basales (Brochu, 1999). Otras especies atribuidas clásicamente a Leidyosuchus han sido incluidas dentro de los géneros Borealosuchus Brochu, 1997, que este autor considera un eusuquio primitivo, y Listrognathosuchus Brochu, 1997, dentro de los Alligatoridae. Brochu (1997) concluye que estos géneros no están estrechamente emparentados con Diplocynodon y no forman un clado. En consecuencia, la familia Leidyosuchidae es un grupo parafilético.

En su análisis filogenético de los Alligatoroidea, Brochu (1999) reúne dentro del género Diplocynodon tres especies: D. rateli, D. darwini y D. hantoniensis. Este trabajo no tiene en cuenta otras especies, como $D$. styriacus y D. tormis. Brochu (1999) define el clado Diplocynodontinae, formado por Diplocynodon y Baryphracta, como "a stem-based monophyletic group including Diplocynodon rateli and all crocodylians more closely related to it than to Alligator mississippiensis".

La distribución estratigráfica conocida de Diplocynodon y formas afines va desde el Paleoceno Superior (Biozona MP6, aff. Diplocynodon sp. de Cernay-Mont-Berru, este de la Cuenca de París) al Mioceno Superior de Bulgaria (descrito como Plioceno medio por Huene y Nikoloff, 1963; véase Ginsburg y Bulot, 1997) y de la Península Ibérica (Buscalioni, 1986b). El material de Diplocynodon más antiguo conocido es de edad Eoceno y proviene del Ypresiense inferior (Cuisiense, MP7) de Le Quesnoy, en Picardía (Nel et al., 1999). Gröessens-Van Dyck (1986) ha descrito dientes de Diplocynodon en el Paleoceno de Bélgica. El material inédito del yacimiento de CernayMont-Berru puede pertenecer a una especie primitiva de Diplocynodon o a un nuevo género. Otro material inédito del Eoceno basal de Le Quesnoy podría representar un nuevo género distinto de Diplocynodon (Hua, en prensa). Esto hace pensar que lo que se ha venido denominando clásicamente Diplocynodon es un linaje de cocodrilos aligatoroideos (Brochu, 1999) y que es posible que varios géneros de aligatoroideos primitivos estén presentes en el Paleoceno-Eoceno de Europa occidental. En este sentido, Brochu (1997) ha especulado con la posibilidad de que el linaje formado por taxones más afines filogenéticamente a Diplocynodon que a los Alligatoridae pueda extenderse hasta el Cretácico superior. Esta hipótesis se basa en la existencia de representantes de los Globidonta, como es el caso de los géneros Brachychampsa, Stangerochampsa y Acynodon, conocidos en el registro fósil desde el Campaniense-Maastrichtiense de Norteamérica y Europa (Norell et al., 1994; Wu et al., 1996; Buscalioni et al., 1999).

Otro punto de controversia son las relaciones de parentesco de Diplocynodon con los Alligatoridae (véase Berg, 1966; Steel, 1973) o los Crocodylidae (véase Kälin, 1955; Ginsburg y Bulot, 1997). En un reciente análisis cladístico, Brochu (1999) ha llegado a la conclusión de que Diplocynodon es un firme representante de los Alligatoroidea, señalando que la oclusión dental de tipo cocodrílido observada por algunos autores (Ginsburg y Bulot, 1997) sólo aparece en formas derivadas ( $D$. styriacus), pero está ausente ancestralmente en Diplocynodon y formas afines.

La taxonomía y filogenia de los "diplocinodontinos" no forman parte de los objetivos de este trabajo. Los resultados de Brochu (1999) apoyan el carácter 
monofilético de los Diplocynodontinae. No obstante, estudios previos han puesto en duda la monofilia de Diplocynodon y han suscitado el problema de reconocer las especies incluidas dentro del género Diplocynodon y aquellas que, aún habiendo sido referidas a Diplocynodon, no pertenecen al mismo y deben ser asignadas a otro género (Buscalioni et al., 1992). Para resolver esta cuestión, es necesario realizar un análisis global incluyendo todas las especies conocidas de Diplocynodon, Baryphracta deponiae y otros aligatoroideos basales.

\section{CONSIDERACIONES TAFONÓMICAS Y PALEOECOLÓGICAS}

A excepción del yacimiento de Cabezo de la Junta, que se localiza en facies microconglomeráticas, todas las demás localidades fosilíferas de las Bardenas se encuentran en facies margosas que, además de fósiles de vertebrados, contienen abundantes restos de fructificaciones de carofitas y gasterópodos, lo que evidencia un medio sedimentario de baja energía (Murelaga, 2000). La fauna acuática está formada por peces, anfibios, tortugas y cocodrilos, lo que sugiere la presencia de un volumen de agua considerable. No obstante, las grietas de desecación y las evidencias de bioturbación observadas en los niveles calizos indican que la masa de agua no era permanente como en un lago y que era poco profunda. En función de estas características, se interpreta que el medio deposicional en el que se dio el reagrupamiento de los restos era un humedal. Esto se aplica a todos los yacimientos de las Bardenas, con excepción del Cabezo de la Junta (Murelaga, 2000). Este último yacimiento parece corresponder a un medio fluvial de mayor energía, debido a la presencia de microconglomerados, una peor conservación de los fósiles y una mayor proporción de restos correspondientes a organismos adémicos (sensu Fernández López, 1990). El bajo grado de meteorización de los fósiles y la presencia de elementos en conexión anatómica parece indicar que los restos de cocodrilos no sufrieron un transporte post-mortal importante. Esta conclusión es válida, asimismo, para los fósiles de otros vertebrados de hábitos acuáticos, como las tortugas. Los datos geológicos y tafonómicos indican que las asociaciones fósiles de las Bardenas se formaron en el centro de una cuenca endorreica, como consecuencia del aporte de agua acumulada en las cordilleras colindantes (Pirineos al NE e Ibérica al SO) y su transporte por arroyos hasta las zonas llanas del centro de la cuenca, donde se generaron diversas masas de agua poco profunda en las cuales se dio una mezcla de restos de formas acuáticas y terrestres (Murelagà, 2000).

Desde un punto de vista paleoecológico, se ha sugerido que las asociaciones fósiles de micromamíferos indican un cambio climático en el límite entre las biozonas Z y A del Rambliense de la Península Ibérica. Los micromamíferos de la biozona $Z$ son típicos de climas secos, mientras que los de la Biozona A indicarían climas más húmedos (López Martínez et al., 1987;
Daams et al., 1997). Este cambio climático también aparece reflejado en el estudio de los cenogramas, rangos de abundancia y curvas de diversidad de los ecosistemas fósiles de las Bardenas, que nos sugieren un clima seco y de campo abierto en la parte superior de la Biozona $\mathrm{Z}$ y un clima más calido, húmedo y con mayores zonas boscosas en la Biozona A (Murelaga, 2000). Estos cambios ambientales son coherentes con los sugeridos a partir del estudio de las asociaciones de ostrácodos de la misma zona, con una transición entre un medio palustre con aportes fluviales de baja salinidad en un clima relativamente cálido (Biozona $\mathrm{Z}$ ) y un medio de aguas alcalinas en un contexto climático más estacional (Biozona A) (Murelaga et al., 1997).

Con todo, no se observan cambios significativos en la composición faunística de los reptiles presentes en los diferentes niveles de las Bardenas (Murelaga, 2000), lo que implica que el cambio medioambiental sugerido a partir del estudio paleoecológico de los mamíferos entre las biozonas $\mathrm{Z}$ y A del Rambliense no fue lo suficientemente drástico como para afectar ecológicamente a los cocodrilos y tortugas. Siguiendo el modelo de los cocodrilos actuales, Markwick (1998) estima que la temperatura es el principal factor condicionante de la distribución global de las antiguas comunidades de cocodrilos. En este sentido, los cocodrilos son excelentes indicadores a la hora de reconstruir paleoclimas. Por su parte, las tortugas son también buenos indicadores paleoclimáticos, pero su distribución siguiendo/los gradientes latitudinales es más amplia que la de los cocodrilos, ya que su rango de restricción térmica es menor. En consecuencia, las tortugas proporcionan un control tafonómico excelente a la hora de analizar la ausencia de registro de cocodrilos debida a condiciones climáticas desfavorables (Markwick, 1998). En conclusión, la presencia conjunta de tortugas y cocodrilos en los yacimientos de las Bardenas evidenciaría un clima relativamente cálido, de tipo subtropical (Murelaga et al., 1999). Los cambios ambientales sugeridos a partir del estudio de los fósiles de mamíferos habrían sido de pequeña magnitud, aún cuando se hubieran dado a escala regional. Otra posibilidad es que tales variaciones reflejen cambios ecológicos ocurridos en áreas relativamente alejadas del centro de la cuenca y correspondientes a los hábitats ocupados por la mayor parte de los mamíferos representados en los yacimientos de las Bardenas (organismos adémicos).

Los cocodrilos ibéricos y europeos del género Diplocynodon persisten al menos hasta el Mioceno Superior (Buscalioni, 1986b; Buscalioni et al., 1992), lo que indica que las condiciones climáticas no se deterioraron drásticamente durante este período de tiempo.

\section{CONCLUSIONES}

Los restos fósiles de cocodrilo del Mioceno Inferior de las Bardenas Reales de Navarra se asignan a una 
especie indeterminada de Diplocynodon, caracterizada por la presencia de una sínfisis mandibular corta. Diplocynodon es el último representante conocido de los aligatoroideos en el continente europeo. Se trata de un cocodrilo generalista de tamaño medio, poco exigente desde el punto de vista térmico, que frecuentaba los medios lacustres o palustres. Los yacimientos de las Bardenas han proporcionado el material más abundante y diverso de Diplocynodon conocido en el Mioceno de la Península Ibérica y el único descrito en detalle hasta la fecha.

Desde un punto de vista paleoambiental, se interpreta que los cocodrilos de las Bardenas habitaban en el medio palustre en el que se acumularon los restos óseos (restos autóctonos o parautóctonos y organismos démicos). La asociación de cocodrilos y tortugas fósiles sugiere un medio ambiente relativamente cálido, de tipo subtropical. No se observan cambios significativos en la composición faunística de los reptiles entre las biozonas $\mathrm{Z}$ y A del Rambliense, lo que sugiere que las fluctuaciones climáticas propuestas a partir del estudio de los mamíferos y ostrácodos no fueron lo suficientemente importantes, en el sentido de provocar notables cambios de temperatura, para desplazar ecológicamente a los cocodrilos u otros reptiles como las tortugas.

\section{AGRADECIMIENTOS}

Nuestro agradecimiento a Eliseo Martínez (Tudela) por su colaboración en la búsqueda de yacimientos fosilíferos en las Bardenas Reales, la familia del Lechuguero de Cascante (Navarra) por su amistad y amable acogida durante nuestros muestreos en la zona de estudio, France de Lapparent de Broin (CNRS, MNHN) por el acceso a las colecciones del MNHN de París y su inestimable ayuda durante el estudio del material fósil y la redacción del manuscrito, Angela D. Buscalioni (Universidad Autónoma de Madrid) por la revisión crítica del manuscrito y todas las sugerencias que han contribuido a mejorarlo, Patxi Ortega (Universidad Autónoma de Madrid) por su ayuda en las primeras fases de estudio del material y Carmen Sesé (Museo de Ciencias Naturales de Madrid-CSIC) por sus amables comentarios. Las fotografías son obra de Imanol Gaztambide y la infografía de la Figura 4 es de Henri Lavina (CNRS, MNHN París). Este trabajo ha sido financiado por un Proyecto de Investigación del Gobierno de Navarra (OF-1994/510) durante el trienio 1995-1997 y un Proyecto de Investigación del Gobierno Vasco entre 1998 y 2000 (PI-1997/53). El primer autor (X.P.S.) ha disfrutado de una Beca de Reincorporación de Doctores del plan I+D del Ministerio de Educación y Ciencia y el segundo autor (X.M.) de una Beca de Formación de Personal Investigador del Gobierno Vasco.

\section{BIBLIOGRAFÍA}

Astibia, H., Ginsburg, L., Janoo, A., Martínez, E., Murelaga, X., Pereda Suberbiola, X. y Sesé, C. 1995. Primeros datos sobre las faunas de tetrápodos fósiles del Terciario continental de las Bardenas Reales de Navarra (Depresión del Ebro). In: XI Jornadas de Paleontología, Tremp, Comunicaciones (Eds. G. López, A. Obrador y E. Vicens), 41-43.

Azanza, B. 1986. Estudio geológico y paleontológico del Mioceno del sector Oeste de Borja (prov. de Zaragoza). Cuadernos de Estudios Borjanos, 17-18, 63-126.

Benton, M.J. and Clark, J.M. 1988. Archosaur phylogeny and the relationships of the Crocodylia. In: The Phylogeny and Classification of the Tetrapods, vol. 1 Amphibians, Reptiles, Birds (Ed. M. J. Benton). Systematics Association Spec. vol. 35 A. Clarendon Press, Oxford, 295-338.

Berg, D.E. 1966. Die Krokodile, insbesondere Asiatosuchus und aff. Sebecus?, aus dem Eozän von Messel bei Darmstadt/Hessem. Abhandlungen des Hessischen Landesamtes für Bodenforschung, 52, 1-105.

Brinkmann, W. und Rauhe, M. 1998. Diplocynodon ratelii Pomel, 1847 (Crocodylia, Leidyosuchidae) aus dem Unter-Oligozän von Céreste (Südfrankreich). Neues Jahrbuch für Geologie und Paläontologie Abh., 209, 295-321.

Brochu, C.A. 1996. Closure of neurocentral sutures during crocodilian ontogeny: implications for maturity assessment in fossil archosaurs. Journal of Vertebrate Paleontology, 16, 49-62.

Brochu, C.A. 1997. A review of "Leidyosuchus" (Crocodyliformes, Eusuchia) from the Cretaceous through Eocene from North America. Journal of Vertebrate Paleontology, 17, 679-697.

Brochu, C.A. 1999. Phylogenetics, taxonomy, and historical biogeography of Alligatoroidea. Journal of Vertebrate Paleontology, 19 (suppl. to 2), 9-100.

Brochu, C.A. 2000. Borealosuchus (Crocodylia) from the Paleocene of Big Bend National Park, Texas. Journal of Paleontology, 74, 181-187.

Buscalioni, A.D. 1986a. Cocodrilos fósiles del registro español. Análisis sistemático y filogenético de la familia Atoposauridae (Reptilia, Crocodylia). Tesis doctoral, Universidad Autónoma de Madrid, 362 pp. (inédita).

Buscalioni, A.D. 1986b. Los cocodrilos fósiles del registro español. Paleontologia i Evolució, 20, 93-98.

Buscalioni, A.D. and Sanz, J.L. 1990. The small crocodile Bernissartia fagessii from the Lower Cretaceous of Galve (Teruel, Spain). Bulletin de l'Institut Royal des Sciences Naturelles de Belgique, 60, 129-150.

Buscalioni, A.D., Sanz, J.L. and Casanovas, M.L. 1992. A new species of the eusuchian crocodile Diplocynodon from the Eocene of Spain. Neues Jahrbuch für Geologie und Paläontologie Abh., 187, 1-29.

Buscalioni, A.D., Ortega, F. and Vasse, D. 1999. The Upper Cretaceous crocodilian assemblage from Laño (Northcentral Spain): implications in the knowledge of the finicretaceous European faunas. Estudios del Museo de Ciencias Naturales de Alava, 14 (Núm. Espec. 1), 213-233.

Clark, J.M. 1994. Patterns of evolution in Mesozoic Crocodyliformes. In: In the shadow of the dinosaurs: early Mesozoic tetrapods (Eds. N.C. Fraser and H.-D. Sues). Cambridge University Press, 84-97. 
Crusafont, M., Truyols, J. y Riba, O. 1966. Contribución al conocimiento de la estratigrafía del Terciario continental de Navarra y Rioja. Notas y Comunicaciones del Instituto Geológico y Minero de España, 90, 53-76.

Cuenca, G., Canudo, J.I., Laplana, C. y Andrés, J.A. 1992. Bio y cronoestratigrafía con mamíferos en la Cuenca Terciaria del Ebro: ensayo de síntesis. Acta Geologica Hispanica, 27, 127-143.

Daams, R. and Freudenthal, M. 1990. The Ramblian and the Aragonian: limits, subdivision, geographical and temporal extension. In: European Neogene Mammal Chronology (Eds. E.H. Lindsay, V. Fahlbusch and P. Mein). Plenum Press, London, 51-59.

Daams, R., Álvarez Sierra, M.A., Meulen, A. Van der y Peláez Campomanes, P. 1997. Los micromamíferos como indicadores de paleoclimas y evolución de las cuencas continentales. In: Registros fósiles e Historia de la Tierra (Eds. E. Aguirre, J. Morales y D. Soria), 281296.

Fabiani, R. 1915. Il Paleogene del Veneto. Memorie dell'Istituto Geologico della R. Università di Padova, $\mathbf{3}$, 1-336.

Fernández López, S. 1990. El significado de la autoctonía/aloctonía tafonómica. Reunión de Tafonomía y Fosilización, Comunicaciones, Madrid, 115-124.

Frey, E., Laemmert, A. und Riess, J. 1987. Baryphracta deponiae n.g. n.sp. (Reptilia, Crocodylia), ein neues Krokodil aus der Grube Messel bei Darmstadt (Hessen, Bundesrepublik Deutschland). Neues Jahrbuch für Geologie und Paläontologie Mon., 1, 15-26.

Gervais, P. 1859. Zoologie et Paléontologie françaises. $2^{\text {ìm }}$ ed., Bertrand Ed., Paris, 544 pp.

Ginsburg, L. et Bulot, C. 1997. Les Diplocynodon (Reptilia, Crocodylia) de l'Orléanien (Miocène inférieur à moyen) de France. Geodiversitas, 19, 107-128.

Grössens-Van Dick, M.C. 1979. Etudes des tortues et des alligators de l'Eocène moyen de Messel conservés au Musée de la ville de Dortmund. Dortmunder Beiträge zur Landeskunde, 12, 79-89.

Grössens-Van Dick, M.C. 1986. Les crocodiliens du gisement "montien" continental de Hainin (Hainaut, Belgique) et leur environment faunique. Annales de la Société Royale Zoologique de Belgique, 116, 55-60.

Hofmann, A. 1885. Crocodiliden aus dem Miocaen der Steiermark. Beiträge zur Paläontologie von ÖsterreichUngarns und des Orients, 5, 26-35.

Hua, S. (en prensa). Les crocodiliens du Sparnacien (Eocène inférieur) du Quesnoy (Oise, France): vers un apport des crocodiliens en tant que marqueurs paléoenvironnementaux. Oryctos.

Huene, F. von und Nikoloff, I. 1963. Ein Pliozänes Krokodil in Bulgarien. Neues Jahrbuch für Geologie und Paläontologie Abh., 118, 266-271.

Kälin, J.A. 1955. Crocodilia. In: Traité de Paléontologie, 5 (Ed. J. Piveteau). Masson, París, 695-784.

Kuhn, O. 1936. Crocodilia. In: Fossilium Catalogus, I: Animalia, 75 (Ed. W. Quenstedt). W. Junk, Den Haag, 1134.

Kuhn, O. 1938. Die Crocodilier aus dem mittleren Eozän des Geiseltales bei Halle. Nova Acta Leopoldina, 6, 313-328.
Laemmert, A. 1993. Dorsal and ventral armour and various positions of embeddings in Diplocynodon (Crocodylia). Kaupia - Darmastädter Beiträge zur Naturgeschichte, $\mathbf{3}$, 35-40.

López Martínez, N., Agustí, J., Cabrera, L., Calvo, J. P., Civis, J., Corrochano, A., Daams, R., Díaz, M., Elizaga, E., Hoyos, M., Martínez, J., Morales, J., Portero, J. M., Robles, F., Santisteban, C. and Torres, T. 1987. Approach to the Spanish continental Neogene synthesis and paleoclimatic interpretation. Proceedings $8^{\text {th }}$ Congress RCMNS, Annales Institutum Geologicum Publicum Hungaricum, 70, 383-391.

Ludwig, R. 1877. Fossile Crocodiliden aus der Tertiärformation des Mainzer Beckens. Palaeontographica, suppl. 3, 1-52.

Lydekker, R. 1887. Note on the Hordwell and other crocodilians. Geological Magazine, (3), 4, 307-312.

Markwick, P.J. 1998. Fossil crocodilians as indicators of Late Cretaceous and Cenozoic climates: implications for using palaeontological data in reconstructing palaeoclimate. Palaeogeography, Palaeoclimatology, Palaeoecology, 137, 205-271.

Martín de Jesús, S., Jiménez Fuentes, E., Fincias, B., Prado, B. del y Mulas Alonso, E. 1987. Los Crocodylia del Eoceno y Oligoceno de la Cuenca del Duero; dientes y osteodermos. Revista Española de Paleontología, 2, 95108.

Mein, P. 1990. Updating of MN Zones. In: European Neogene Mammal Chronology (Eds. E.H. Lindsay, V. Fahlbusch and P. Mein). NATO ASI Series, (A) 180. Plenum Press, London, 73-90.

Michard, J.-G., Broin, F. de, Brunet, M. et Hell, J. 1990. Le plus ancien crocodilien néosuchien spécialisé à caractères "eusuchiens" du continent africain (Crétacé inférieur, Cameroun). Comptes Rendus de l'Académie des Sciences, Paris, 311 (II), 365-371.

Murelaga, X. 2000. Estudio de las faunas de vertebrados del Mioceno inferior de las Bardenas Reales y áreas colindantes (Cuenca del Ebro, Navarra). Tesis doctoral, Facultad de Ciencias, Universidad del País Vasco/EHU, Bilbao, 363 pp. (inédita).

Murelaga, X., Caballero, F., Rodríguez Lázaro, J., Astibia, H. y Pereda Suberbiola, X. 1997. Análisis preliminar de los ostrácodos del Mioceno inferior de las Bardenas Reales de Navarra (Cuenca del Ebro). Geogaceta, 22, 129-132.

Murelaga, X., Lapparent de Broin, F. de, Pereda Suberbiola, X. et Astibia, H. 1999. Deux nouvelles espèces de chéloniens dans le Miocène inférieur du bassin de l'Ebre (Bardenas Reales de Navarre). Comptes Rendus de l'Académie des Sciences, Paris, 328 (IIa), 423-429.

Nel, A., Plöeg, G. de, Dejax, J., Dutheil, D., Franceschi, D. de, Gheerbrant, E., Godinot, M., Hervet, S., Menier, J.-J., Augé, M., Bignot, G., Cavagnetto, C., Duffaud, S., Gaudant, J., Hua, S., Jossang, A., Lapparent de Broin, F. de, Pozzi, J.-P., Paicheler, J.-C., Beuchet, F. et Rage, J.C. 1999. Un gisement sparnacien exceptionnel à plantes, arthropodes et vertébrés (Eocène basal, MP7): Le Quesnoy (Oise, France). Comptes Rendus de l'Académie des Sciences, Paris, 329 (IIa), 65-72. 
Norell, M.A. and Clark, J.M. 1990. A reanalysis of Bernissartia fagesii, with comments on its phylogenetic position and its bearing on the origin and diagnosis of the Eusuchia. Bulletin de l'Institut Royal des Sciences naturelles de Belgique, Sc. Terre, 60, 115-128.

Norell, M.A., Clark, J.M. and Hutchinson, J.H. 1994. The Late Cretaceous alligatoroid Brachychampsa montana (Crocodylia): new material and putative relationships. American Museum Novitates, 3116, 1-26.

Ortega, F. 1990. Descripción anatómica de fragmentos craneales y mandibulares de Diplocynodon Pomel, 1847 (Crocodylia, Alligatoridae) del Paleógeno de la Cuenca del Duero. Discusión sistemática. Tesis de licenciatura, Departamento de Geología, Universidad de Salamanca, 161 pp. (inédita).

Pereda Suberbiola, X., Murelaga, X. y Astibia, H. 1996. Cocodrilos y tortugas del Mioceno de las Bardenas Reales de Navarra (Depresión del Ebro). In: XII Jornadas de Paleontología, Badajoz, Comunicaciones (Eds. T. Palacios y R. Gozalo), 99-101.

Pomel, A. 1847. Note sur les animaux fossiles découverts dans le Départment de l'Allier. Bulletin de la Société géologique de France, (II), 4, 378-385.

Pomel, A. 1853. Catalogue méthodique et descriptif des vertébrés fossiles dévouverts dans le bassin hydrographique supérieur de la Loire et surtout dans la vallée de son affluent principal, l'Allier. Ed. J.B. Baillère, Paris, 193 pp.

Rauhe, M. and Rossmann, T. 1995. News about fossil crocodiles from the middle Eocene of Messel and Geiseltal, Germany. Hallesches Jahrbuch für Geowissenchaften, 17, 81-92.

Steel, R. 1973. Crocodylia. In: Handbuch der Paläoherpetologie, vol. 16. G. Fischer-Verlag, Stuttgart, 116 pp.

Vaillant, L. 1872. Étude zoologique sur les crocodiliens fossiles tertiaires de Saint-Gérand-le-Puy. Annales des Sciences géologiques, 3, 1-58.

Vignaud, P., Brunet, M. et Jehenne, Y. 1996. Un crâne de Diplocynodon (Crocodylomorpha, Alligatoridae) de l'Oligocène inférieur de Dordogne (France). Comptes Rendus de l'Académie des Sciences, Paris, 322 (IIa), 595-601.

Wood, S. 1846. On the discovery of an Alligator and of several new Mammalia in the Hordwell Cliff; with observations upon the Geological Phenomena of that locality. London Geological Journal, 1, 1-7.

Wu, X.-C., Brikmann, D.B. and Russell, A.P. 1996. A new alligator from the Upper Cretaceous of Canada and the relationships of the early eusuchians. Palaeontology, 39, 351-375. 